\title{
Nonlinear Feedback Controllers and Compensators: A State-Dependent Riccati Equation Approach
}

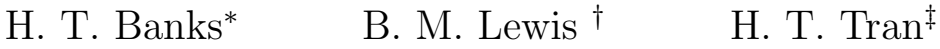 \\ Department of Mathematics \\ Center for Research in Scientific Computation \\ North Carolina State University \\ Raleigh, NC 27695
}

\begin{abstract}
State-dependent Riccati equation (SDRE) techniques are rapidly emerging as general design and synthesis methods of nonlinear feedback controllers and estimators for a broad class of nonlinear regulator problems. In essence, the SDRE approach involves mimicking standard linear quadratic regulator (LQR) formulation for linear systems. In particular, the technique consists of using direct parameterization to bring the nonlinear system to a linear structure having state-dependent coefficient matrices. Theoretical advances have been made regarding the nonlinear regulator problem and the asymptotic stability properties of the system with full state feedback. However, there have not been any attempts at the theory regarding the asymptotic convergence of the estimator and the compensated system. This paper addresses these two issues as well as discussing numerical methods for approximating the solution to the SDRE. The Taylor series numerical methods works only for a certain class of systems, namely with constant control coefficient matrices, and only in small regions. The interpolation numerical method can be applied globally to a much larger class of systems. Examples will be provided to illustrate the effectiveness and potential of the SDRE technique for the design of nonlinear compensator-based feedback controllers.
\end{abstract}

\section{Introduction}

Linear quadratic regulation (LQR) is a well established, accepted, and effective theory for the synthesis of control laws for linear systems. However, most mathematical models for biological systems, including HIV dynamics with immune response [4, 17], as well as those for physical processes, such as those arising in the microelectronic industry [3] and satellite dynamics [22], are inherently nonlinear. A number of methodologies exist for the control design and synthesis of these highly nonlinear systems. These techniques include a large number of linear design methodologies $[33,15]$ such as Jacobian linearization and feedback linearization used in conjunction with gain scheduling [25]. Nonlinear design techniques have also been proposed including dynamic inversion [27], recursive backstepping [18], sliding mode control [27], and adaptive control [18]. In addition, other nonlinear controller designs such as methods based on estimating the solution of the HamiltonJacobi-Bellman (HJB) equation can be found in a comprehensive review article [5]. Each of these

\footnotetext{
${ }^{*}$ htbanks@eos.ncsu.edu

${ }^{\dagger}$ bmlewis2@unity.ncsu.edu

†ran@control.math.ncsu.edu
} 
techniques has its set of tuning rules that allow the modeler and designer to make trade-offs between control effort and output error. Other issues such as stability and robustness with respect to parameter uncertainties and system disturbances are also features that differ depending on the control methodology considered.

One of the highly promising and rapidly emerging methodologies for designing nonlinear controllers is the state-dependent Riccati equation (SDRE) approach in the context of the nonlinear regulator problem. This method, which is also referred to as the Frozen Riccati Equation (FRE) method [11], has received considerable attention in recent years $[9,10,12,26]$. In essence, the SDRE method is a systematic way of designing nonlinear feedback controllers that approximate the solution of the infinite time horizon optimal control problem and can be implemented in realtime for a broad class of applications. Through extensive numerical simulations, the SDRE method has demonstrated its effectiveness as a technique for, among others, controlling an artificial human pancreas [23], for the regulation of the growth of thin films in a high-pressure chemical vapor deposition reactor [3, 2, 30], and for the position and attitude control of a spacecraft [28]. More specifically, recent articles $[3,2]$ have reported on the successful use of SDRE in the development of nonlinear feedback control methods for real-time implementation on a chemical vapor deposition reactor. The problems are optimal tracking problems (for regulation of the growth of thin films in a high-pressure chemical vapor deposition (HPCVD) reactor) that employ state-dependent Riccati gains with nonlinear observations and the resulting dual state-dependent Riccati equations for the compensator gains.

Even though these computational efforts are very promising, the present investigation opens a host of fundamental mathematical questions that should provide a rich source of theoretical challenges. In particular, much of the focus thus far has been on the full state feedback theory, the implementation of the method, and numerical methods for solving the SDRE with a constant control coefficient matrix. In most cases, the theory developed also involves using nonlinear weighting coefficients for the state and control in the cost functional to produce near optimal solutions. This methodology is quite useful and also quite difficult to implement for complex systems. Therefore, it is of general interest to explore the use of constant weighting matrices to produce a suboptimal control law that has the advantage of ease of configuration and implementation. In addition, the development of a comprehensive theory is needed for approximation and convergence of the statedependent Riccati equation approach for nonlinear compensation. Finally, a current approach for solving the SDRE is via symbolic software package such as Macsyma or Mathematica [9]. However, this is only possible for systems having special structures. In [6], an efficient computational methodology was proposed that requires splitting the state-dependent coefficient matrix $A(x)$ into a constant matrix part and a state-dependent part as $A(x)=A_{0}+\varepsilon \Delta A(x)$. This method is effective locally for systems with constant control coefficients and if the function $\Delta A(x)$ is not too complicated (e.g., when it has the same function of $x$ in all entries) then the SDRE can be solved through a series of constant-valued matrix Lyapunov equations. The assumption on the form of $\Delta A(x)$, however, does limit the problems for which this SDRE approximation method is applicable. Another method, based on interpolation, is effective for nonconstant control coefficients and it can be applied throughout the state space. The interpolation approach involves varying the SDRE over the states and creating a grid of values for the control $u(x)$ or the solution to the SDRE $\Pi(x)$.

In this paper, we examine the SDRE technique with constant weighting coefficients. In Section 2, we review the full state feedback theory and prove local asymptotic stability for the closed loop system. A simple example with an attainable exact solution is presented to verify the theoretical result. This example also exhibits the efficiency of the method outside of the region for which the condition in the proof predicts asymptotic stability. Section 3 summarizes two of the numerical methods that are currently available for the approximation of the SDRE solution. Sec- 
tion 4 presents the extension of the SDRE methodology to the nonlinear state estimation problem. It includes local convergence results of the nonlinear state estimator and a numerical example. Section 5 addresses the estimator based feedback control synthesis including a local asymptotic stability result for the closed loop system as well as an illustrative example.

\section{$2 \quad$ Full State Response}

In this section we formulate the optimal control problem where it is assumed that all state variables are available for feedback. In [9], the theory for full state feedback is developed for nonconstant weighting matrices. Here, we formulate our optimal control problem with constant weighting matrices. In particular, the cost functional is given by the integral

$$
J\left(x_{0}, u\right)=\frac{1}{2} \int_{t_{0}}^{\infty} x^{T} Q x+u^{T} R u d t
$$

where $x \in \Re^{n}, u \in \Re^{m}, Q \in \Re^{n \times n}$ is symmetric positive semidefinite (SPSD), and $R \in \Re^{m \times m}$ is symmetric positive definite (SPD). Associated with the performance index (1) are the nonlinear dynamics

$$
\dot{x}=f(x)+B(x) u,
$$

where $f(x)$ is a nonlinear function in $C^{k}$ and $B(x) \in \Re^{n \times m}$ is a matrix-valued function. Rewriting the nonlinear dynamics (2) in the state-dependent coefficient (SDC) form $f(x)=A(x) x$, we have

$$
\dot{x}=A(x) x+B(x) u,
$$

where, in general, $A(x)$ is unique only if $x$ is scalar [9]. For the multivariable case we consider an illustrative example, $f(x)=\left[x_{2}, x_{1}^{3}\right]^{T}$. The obvious SDC parameterization is

$$
A_{1}(x)=\left[\begin{array}{cc}
0 & 1 \\
x_{1}^{2} & 0
\end{array}\right]
$$

However, we can find another SDC parameterization

$$
A_{2}(x)=\left[\begin{array}{cc}
x_{2} / x_{1} & 0 \\
x_{1}^{2} & 0
\end{array}\right]
$$

by dividing and multiplying each component of $f(x)$ by $x_{1}$. We find yet another SDC parameterization

$$
A_{3}(x)=\left[\begin{array}{cc}
-x_{2} & 1+x_{1} \\
x_{1}^{2} & 0
\end{array}\right]
$$

by adding and subtracting the term $x_{1} x_{2}$ to $f_{1}(x)$. Since there exists at least two SDC parameterizations, there are an infinite number. This is true since for all $0 \leq \alpha \leq 1$,

$$
\alpha A_{1}(x) x+(1-\alpha) A_{2}(x) x=\alpha f(x)+(1-\alpha) f(x)=f(x) .
$$

Remark 2.1. Choosing the SDRE parameterization

Because of the many available SDC parameterizations, as we design the control law we must choose the one that is most appropriate for the system and control objectives of 
interest. One factor that is of considerable importance is the state-dependent controllability matrix (or in estimation theory, the state-dependent observability matrix). As in the linear theory, the matrix is given by

$$
M(x)=\left[\begin{array}{llll}
B(x) & A(x) B(x) & \ldots & A^{(n-1)}(x) B(x)
\end{array}\right] .
$$

In linear system theory, if $M$ (a constant in this case) has full rank then the system is controllable. In the nonlinear case we must seek a parameterization that gives $M(x)$ full rank for the entire domain for which the system is to be controlled. For estimation (to be considered in Section 4), one must consider the state-dependent observability matrix,

$$
O(x)=\left[\begin{array}{c}
C(x) \\
C(x) A(x) \\
\vdots \\
C(x) A^{n-1}(x)
\end{array}\right]
$$

when choosing the parameterization.

The Hamiltonian for the optimal control problem (1)-(3) is given by

$$
H(x, u, \lambda)=\frac{1}{2}\left(x^{T} Q x+u^{T} R u\right)+\lambda^{T}(A(x) x+B(x) u) .
$$

From the Hamiltonian, the necessary conditions for the optimal control are found to be

$$
\begin{aligned}
& \dot{\lambda}=-Q x-\left[\frac{\partial(A(x) x)}{\partial x}\right]^{T} \lambda-\left[\frac{\partial(B(x) u)}{\partial x}\right]^{T} \lambda, \\
& \dot{x}=A(x) x+B(x) u,
\end{aligned}
$$

and

$$
0=R u+B^{T}(x) \lambda
$$

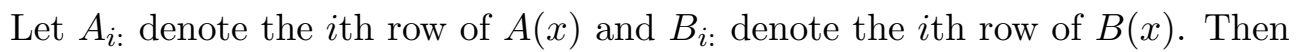

$$
\begin{aligned}
\frac{\partial(A(x) x)}{\partial x} & =A(x)+\frac{\partial(A(x))}{\partial x} x \\
& =A(x)+\left[\begin{array}{ccc}
\frac{\partial A_{1:}}{\partial x_{1}} x & \ldots & \frac{\partial A_{1:}}{\partial x_{n}} x \\
\vdots & \ddots & \vdots \\
\frac{\partial A_{n:}}{\partial x_{1}} x & \ldots & \frac{\partial A_{n:}}{\partial x_{n}} x
\end{array}\right]
\end{aligned}
$$

and

$$
\frac{\partial(B(x) u)}{\partial x}=\left[\begin{array}{ccc}
\frac{\partial B_{1:}}{\partial x_{1}} u & \ldots & \frac{\partial B_{1:}}{\partial x_{n}} u \\
\vdots & \ddots & \vdots \\
\frac{\partial B_{n:}}{\partial x_{1}} u & \ldots & \frac{\partial B_{n:}}{\partial x_{n}} u
\end{array}\right] .
$$

Mimicking the Sweep Method [19], the co-state is assumed to be of the form $\lambda=\Pi(x) x$ (note the state dependency). Using this form for $\lambda$ in equation (8) we obtain a feedback control $u=$ $-R^{-1} B^{T}(x) \Pi(x) x$. Substituting this control back into (7), we find $\dot{x}=A(x) x-B(x) R^{-1} B^{T}(x) \Pi(x) x$. 
To find the matrix-valued function $\Pi(x)$, we differentiate $\lambda=\Pi(x) x$ with respect to time along a trajectory to obtain

$$
\begin{aligned}
\dot{\lambda} & =\dot{\Pi}(x) x+\Pi(x) \dot{x} \\
& =\dot{\Pi}(x) x+\Pi(x) A(x) x-\Pi(x) B(x) R^{-1} B^{T}(x) \Pi(x) x,
\end{aligned}
$$

where we use the notation

$$
\dot{\Pi}(x)=\sum_{i=1}^{n} \Pi_{x_{i}}(x) \dot{x}_{i}(t) .
$$

If we set this equal to $\dot{\lambda}$ from (6), we find

$$
\begin{aligned}
\dot{\Pi}(x) x & +\Pi(x) A(x) x-\Pi(x) B(x) R^{-1} B^{T}(x) \Pi(x) x \\
& =-Q x-\left[A(x)+\frac{\partial(A(x))}{\partial x} x\right]^{T} \Pi(x) x-\left[\frac{\partial(B(x) u)}{\partial x}\right]^{T} \Pi(x) x .
\end{aligned}
$$

Rearranging terms we find

$$
\begin{aligned}
{[(\dot{\Pi}(x)+} & {\left.\left[\frac{\partial(A(x))}{\partial x}\right]^{T} \Pi(x)+\left[\frac{\partial(B(x) u)}{\partial x}\right]^{T} \Pi(x)\right) } \\
& \left.+\left(\Pi(x) A(x)+A^{T}(x) \Pi(x)-\Pi(x) B(x) R^{-1} B^{T}(x) \Pi(x)+Q\right)\right] x=0 .
\end{aligned}
$$

If we assume that $\Pi(x)$ solves the SDRE, which is given by

$$
\Pi(x) A(x)+A^{T}(x) \Pi(x)-\Pi(x) B(x) R^{-1} B^{T}(x) \Pi(x)+Q=0,
$$

then the following condition must be satisfied for optimality:

$$
\dot{\Pi}(x)+\left[\frac{\partial(A(x))}{\partial x}\right]^{T} \Pi(x)+\left[\frac{\partial(B(x) u)}{\partial x}\right]^{T} \Pi(x)=0 .
$$

To be consistent with [9], we will refer to (13) as the Optimality Criterion. The suboptimal control for (1) and (3) is found by solving (12) for $\Pi(x)$. Then, the optimal control problem has the suboptimal solution

$$
u=-K(x) x \quad \text { where } \quad K(x)=R^{-1} B^{T}(x) \Pi(x) .
$$

In [9], a methodology is presented for forming a state-dependent weighting matrix $Q(x)$ so as to find an optimal control solution. This methodology is useful but somewhat difficult for complex systems. Therefore, we focus on the suboptimal control law that is useful for all systems and has the benefit of ease of implementation. The focus for the remainder of the section will be the local asymptotic convergence of the state using the SDRE solution to formulate the suboptimal control. Some of the nomenclature associated with the SDC parameterization is now given to aid in the presentation of our theoretical results.

Definition 2.1 (Detectable Parameterization). $A(x)$ is a detectable parameterization of the nonlinear system in $\Omega$ if the pair $\left(Q^{1 / 2}, A(x)\right)$ is detectable for all $x \in \Omega$.

Definition 2.2 (Stabilizable Parameterization). $A(x)$ is a stabilizable parameterization of the nonlinear system in $\Omega$ if the pair $(A(x), B(x))$ is stabilizable for all $x \in \Omega$. 
We now present a lemma relating the linearization of the original system about the origin and the SDC parameterization evaluated at zero. To motivate the lemma, consider the following simple example:

$$
\dot{x}=f(x)=\left[\begin{array}{c}
x_{1} x_{2} \sin \left(x_{2}\right)+x_{2} \cos \left(x_{1}\right) \\
x_{1}^{2}+2 x_{2}
\end{array}\right] .
$$

Two obvious choices for SDC parameterizations of this system are

$$
A_{1}(x)=\left[\begin{array}{cc}
x_{2} \sin \left(x_{2}\right) & \cos \left(x_{1}\right) \\
x_{1} & 2
\end{array}\right]
$$

and

$$
A_{2}(x)=\left[\begin{array}{cc}
0 & x_{1} \sin \left(x_{2}\right)+\cos \left(x_{1}\right) \\
x_{1} & 2
\end{array}\right] .
$$

The gradient of $f(x)$ is given by

$$
\nabla f(x)=\left[\begin{array}{cc}
x_{2} \sin \left(x_{2}\right)-x_{2} \sin \left(x_{1}\right) & x_{1} \sin \left(x_{2}\right)+x_{1} x_{2} \cos \left(x_{2}\right)+\cos \left(x_{1}\right) \\
2 x_{1} & 2
\end{array}\right] .
$$

Since the linearization of $f$ at the origin is the gradient evaluated at zero, we obtain

$$
A_{1}(0)=A_{2}(0)=\nabla f(0)=\left[\begin{array}{ll}
0 & 1 \\
0 & 2
\end{array}\right] \text {. }
$$

Generalizing this result, we have that:

Lemma 2.1. For any $S D C$ parameterization $A(x) x, A(0)$ is the linearization of $f(x)$ about the zero equilibrium.

Proof. Let $A_{1}(x)$ and $A_{2}(x)$ be two distinct parameterizations of $f(x)$ and let $\tilde{A}(x)=A_{1}(x)-A_{2}(x)$. Then $\tilde{A}(x) x=0$ for all $x$ and

$$
\frac{\partial \tilde{A}(x) x}{\partial x}=\tilde{A}(x)+\frac{\partial \tilde{A}(x)}{\partial x} x=0 .
$$

Then, because the second term on the right is zero at $x=0$, it follows that $\tilde{A}(0)=0$ which implies $A_{1}(0)=A_{2}(0)$. Therefore we have that the parameterization evaluated at zero is unique. Without loss of generality, consider the parameterization given by $A_{1}(x)$. The linearized system is

$$
\dot{z}=\nabla f(0) z .
$$

But

$$
\nabla f(x)=A_{1}(x)+\frac{\partial A_{1}(x)}{\partial x} x
$$

so $\nabla f(0)=A_{1}(0)$ which has been shown to be unique for all parameterizations.

Remark 2.2. It is assumed that the solution to the SDRE (12) exists for all $x$ in the neighborhood of the origin being considered (and, naturally, $(A(x), B(x))$ is a stabilizable parameterization). 
Thus, it is of logical consequence that the solution exists at $x=0$ and that $\Pi_{0}=\Pi(0)$ solves the linear system algebraic Riccati equation (ARE)

$$
\Pi_{0} A_{0}+A_{0}^{T} \Pi_{0}-\Pi_{0} B_{0} R^{-1} B_{0}^{T} \Pi_{0}+Q=0,
$$

with $A_{0}=A(0)$ (here, $A_{0}$ is uniquely defined rather than in [7], where $A_{0}$ is not necessarily $A(0)$ ) and $B_{0}=B(0)$. Thus, a natural and useful representation of the matrix $\Pi(x)$ is the representation

$$
\Pi(x)=\Pi_{0}+\Delta \Pi(x),
$$

where $\Delta \Pi(x)=\Pi(x)-\Pi(0)$ and $\Delta \Pi(0)=0$. Likewise, $A(x)$ and $B(x)$ can be rewritten using the constant matrices $A_{0}$ and $B_{0}$ and nonconstant matrices $\Delta A(x)$ and $\Delta B(x)$ (defined in a way similar to $\Delta \Pi(x))$ as

$$
A(x)=A_{0}+\Delta A(x),
$$

and

$$
B(x)=B_{0}+\Delta B(x),
$$

with $\Delta A(0)=0$ and $\Delta B(0)=0$. This leads to the control $u$ being represented as a the sum of a constant matrix and an incremental matrix,

$$
u(x)=-\left(K_{0}+\Delta K(x)\right) x,
$$

where

$$
K_{0}=R^{-1} B_{0}^{T} \Pi_{0}
$$

and

$$
\Delta K(x)=R^{-1}\left(B^{T}(x) \Delta \Pi(x)+\Delta B^{T}(x) \Pi_{0}\right) .
$$

By construction $\Delta \Pi(x)$ and $\Delta B(x)$ are zero at the origin so that $\Delta K(0)=0$. Under continuity assumptions on $A(x)$ and $B(x)$, along with the assumption that the SDC parameterization is a detectable and stabilizable parameterization, it follows that $\Pi(x)$ is continuous. This follows from (see page $315,[24]$ )

Theorem 2.1. The maximal hermitian solution $\Pi$ of the constant ARE

$$
\Pi A+A^{T} \Pi-\Pi B R^{-1} B^{T} \Pi+Q=0
$$

is a continuous function of $w=\left(B R^{-1} B^{T}, A, Q\right)$.

Hence, since we require that $A(x)$ and $B(x)$ are continuous, it can be concluded that the solution to the SDRE, $\Pi(x)$, is also continuous. Therefore, the norms the incremental matrices $\Delta A(x), \Delta B(x)$, and $\Delta K(x)$ are small in a neighborhood of zero. For the remainder of this chapter, we denote the $\epsilon$-ball around $z$ as

$$
\mathcal{B}_{\epsilon}(z)=\{x:\|x-z\|<\epsilon\} .
$$

With the use of the ideas in the above discussions, the following theorem can be proven:

Theorem 2.2. Assume that the system

$$
\dot{x}=f(x)+B(x) u
$$

is such that $f(x)$ and $\frac{\partial f(x)}{\partial x_{j}}(j=1, \ldots, n)$ are continuous in $x$ for all $\|x\| \leq \hat{r}, \hat{r}>0$, and that $f(x)$ can be written as $f(x)=A(x) x$ (in SDC form). Assume further that $A(x)$ and $B(x)$ are continuous and the system defined by (1) and (3) is a detectable and stabilizable parameterization in some nonempty neighborhood of the origin $\Omega \subseteq \mathcal{B}_{\hat{r}}(0)$. Then the system with the control given by (14) is locally asymptotically stable. 
Proof. Let $r>0$ be the largest radius such that $\mathcal{B}_{r}(0) \subseteq \Omega$. By the assumption that the system is stabilizable at $x=0$ we can use LQR theory to create a matrix $K_{0}$ such that all eigenvalues of $A_{0}-B_{0} K_{0}$ have negative real parts. This implies the existence of $\beta>0$ such that $\operatorname{Re}(\lambda)<-\beta$ for all eigenvalues $(\lambda)$ of $A_{0}-B_{0} K_{0}$. Using the maps defined by (17), (18), and (19), we have that the controlled nonlinear dynamics can be rewritten in the form

$$
\begin{aligned}
\dot{x} & =A(x) x-B(x) K(x) x \\
& =\left(A_{0}+\Delta A(x)\right) x-\left(B_{0}+\Delta B(x)\right)\left(K_{0}+\Delta K(x)\right) x \\
& =\left(A_{0}-B_{0} K_{0}\right) x+\left(\Delta A(x)-B(x) \Delta K(x)-\Delta B(x) K_{0}\right) x .
\end{aligned}
$$

If we let

$$
g(x)=\Delta A(x)-B(x) \Delta K(x)-\Delta B(x) K_{0}
$$

and $h(x)=g(x) x$ then the system is given by

$$
\dot{x}=\left(A_{0}-B_{0} K_{0}\right) x+h(x) .
$$

Examination of $h(x)$ reveals that we are dealing with an almost linear system satisfying the property

$$
\lim _{\|x\| \rightarrow 0} \frac{\|h(x)\|}{\|x\|}=0
$$

This is easily deduced from the inequality

$$
\|h(x)\| \leq\|g(x)\|\|x\| \leq\left(\|\Delta A(x)\|+\|B(x)\|\|\Delta K(x)\|+\|\Delta B(x)\|\left\|K_{0}\right\|\right)\|x\|,
$$

where the norms of the incremental matrices are known to be continuous and equal to zero at the origin. Since $B(x)$ is continuous, the norm of $B(x)$ is bounded for any bounded region. Hence, $\|g(x)\| \rightarrow 0$ as $\|x\| \rightarrow 0$ and, hence, $h(x)$ satisfies condition (24). From theoretical results on almost linear systems [8], we know that if the eigenvalues of $A_{0}-B_{0} K_{0}$ have negative real parts, $h(x)$ is continuous around the origin, and condition (24) holds, then $x=0$ is asymptotically stable. We outline the known proof of this result for our specific system since it will serve as a template for the subsequent arguments presented below.

Let $\zeta>0$ be given. Then, there exists a $\hat{\delta} \in(0, r)$ such that $\|h(x)\| \leq \zeta\|x\|$ whenever $\|x\| \leq \hat{\delta}$. Let $x(0)=x_{0} \in \mathcal{B}_{\hat{\delta}}(0)$. By the assumptions on $f$ and by continuity, the solution exists and remains in $\mathcal{B}_{\hat{\delta}}(0)$ at least until some time $\hat{t}>0$. For $t \in[0, \hat{t})$ we can then express the solution to (23) using the variation of constants formula to obtain

$$
x(t)=e^{\left(A_{0}-B_{0} K_{0}\right) t} x(0)+\int_{0}^{t} e^{\left(A_{0}-B_{0} K_{0}\right)(t-s)} h(x(s)) d s .
$$

Taking the norm of both sides we have

$$
\|x(t)\| \leq\left\|e^{\left(A_{0}-B_{0} K_{0}\right) t}\right\|\|x(0)\|+\zeta \int_{0}^{t}\left\|e^{\left(A_{0}-B_{0} K_{0}\right)(t-s)}\right\|\|x(s)\| d s
$$

for as long as $\|x(t)\|<\hat{\delta}$. There exists a positive constant $G$ such that

$$
\left\|e^{\left(A_{0}-B_{0} K_{0}\right) t}\right\| \leq G e^{-\beta t}
$$

so that

$$
\|x(t)\| \leq G e^{-\beta t}\|x(0)\|+\zeta G \int_{0}^{t} e^{-\beta(t-s)}\|x(s)\| d s .
$$


Multiplying by $e^{\beta t}$ and invoking the Gronwall inequality, we find

$$
e^{\beta t}\|x(t)\| \leq G\|x(0)\| e^{\zeta G t}
$$

or

$$
\|x(t)\| \leq G\|x(0)\| e^{-(\beta-\zeta G) t}
$$

for all $t>0$ such that $\|x(t)\|<\hat{\delta}$. We restrict our initial condition domain further by selecting $\zeta \in(0, \beta / G)$. Then, with $\hat{\delta}$ corresponding to this $\zeta$, given $\epsilon \in(0, \hat{\delta}]$ we select $\delta=\min \{\hat{\delta}, \epsilon / G\}$. Then, for $x_{0} \in \mathcal{B}_{\delta}(0)$, we have that $\|x(t)\|<\epsilon \leq \hat{\delta}$ for all $t>0$ and $x=0$ is stable. Moreover, (25) holds for all $t>0$ if $x_{0} \in \mathcal{B}_{\delta}(0)$. Since $\beta-\zeta G>0$ we have $x=0$ is in fact asymptotically stable.

\subsection{Example 1: Exact Solution}

In this section we consider a simple example from [20] that has an exact solution. This example is of particular interest because the numerical method in [7] failed to produce a stabilizing control based on the approximate SDRE solution. The cost functional for the example under consideration is

$$
J\left(x_{0}, u\right)=\int_{0}^{\infty}\left(x^{T}\left[\begin{array}{cc}
\frac{1}{2} & 0 \\
0 & \frac{1}{2}
\end{array}\right] x+\frac{1}{2} u^{2}\right) d t
$$

with associated nonlinear state dynamics

$$
\left[\begin{array}{c}
\dot{x}_{1} \\
\dot{x}_{2}
\end{array}\right]=\left[\begin{array}{l}
x_{2} \\
x_{1}^{3}
\end{array}\right]+\left[\begin{array}{l}
0 \\
1
\end{array}\right] u
$$

An SDC parameterization is given by

$$
\left[\begin{array}{c}
\dot{x}_{1} \\
\dot{x}_{2}
\end{array}\right]=\left[\begin{array}{cc}
0 & 1 \\
x_{1}^{2} & 0
\end{array}\right]\left[\begin{array}{l}
x_{1} \\
x_{2}
\end{array}\right]+\left[\begin{array}{l}
0 \\
1
\end{array}\right] u .
$$

The resulting constant and incremental matrices (17) and (18) have the form

$$
A_{0}=\left[\begin{array}{ll}
0 & 1 \\
0 & 0
\end{array}\right], \quad \Delta A(x)=\left[\begin{array}{cc}
0 & 0 \\
x_{1}^{2} & 0
\end{array}\right],
$$

$B_{0}=B$, and $\Delta B(x)=0$. This parameterization has state-dependent controllability matrix given by

$$
M(x)=\left[\begin{array}{ll}
0 & 1 \\
1 & 0
\end{array}\right]
$$

which has full rank for all $x \in \Re^{2}$. Therefore, the SDC parameterization is such that $(A(x), B(x))$ is controllable for all $x$ and $\left(Q^{1 / 2}, A(x)\right)$ is observable for all $x$. Hence, we can assume that the SDRE solution can be used to construct a locally stabilizing suboptimal control via (14). The SDRE for this SDC parameterization is given by

$$
\Pi\left[\begin{array}{cc}
0 & 1 \\
x_{1}^{2} & 0
\end{array}\right]+\left[\begin{array}{cc}
0 & x_{1}^{2} \\
1 & 0
\end{array}\right] \Pi-\Pi\left[\begin{array}{l}
0 \\
1
\end{array}\right] 2\left[\begin{array}{ll}
0 & 1
\end{array}\right] \Pi+\left[\begin{array}{cc}
\frac{1}{2} & 0 \\
0 & \frac{1}{2}
\end{array}\right]=0
$$

where $\Pi(x)$ is a symmetric matrix. The solution to the SDRE has the form

$$
\Pi(x)=\left[\begin{array}{cc}
\sqrt{x_{1}^{4}+1}\left(\sqrt{\frac{x_{1}^{2}+\sqrt{x_{1}^{4}+1}}{2}+\frac{1}{4}}\right) & \frac{x_{1}^{2}+\sqrt{x_{1}^{4}+2}}{2} \\
\frac{x_{1}^{2}+\sqrt{x_{1}^{4}+2}}{2} & \sqrt{\frac{x_{1}^{2}+\sqrt{x_{1}^{4}+1}}{2}+\frac{1}{4}}
\end{array}\right] .
$$


By setting the state to zero we find that the solution to the SDRE at the origin (15) is

$$
\Pi_{0}=\left[\begin{array}{ll}
\frac{\sqrt{3}}{2} & \frac{\sqrt{2}}{2} \\
\frac{\sqrt{2}}{2} & \frac{\sqrt{3}}{2}
\end{array}\right]
$$

and $\Delta \Pi(x)=\Pi(x)-\Pi_{0}$. Since $\Delta B(x)=0, \Delta K(x)=R^{-1} B^{T} \Delta \Pi(x)$ and we have that $g(x)=$ $\|\Delta A(x)\|+\|B\|\|\Delta K(x)\|$. For this example, we find that the eigenvalues of the closed loop system are $-0.866 \pm 0.5 i$. In Figure 1(a) we see that if we let $G=4$ and $\beta=0.866$ then we have found a bound of the form $\left\|e^{\left(A_{0}-B_{0} K_{0}\right) t}\right\| \leq G e^{-\beta t}$. Figure 1(b) is a plot of

$$
y=4 g(x)-|\operatorname{Re}(\lambda)|
$$

vs. $x_{1}$ (since $\Delta A(x)$ and $\Delta K(x)$ are both dependent only on $x_{1}$ ) where we have used the $\|\cdot\|_{2}$ norm for $g$. This gives us an idea of the region from which we can choose initial conditions so that $-(\beta-\zeta G)<0$. By approximating the zeros of $y$ we see that $\left|x_{1}\right| \leq 0.31$ produces negative values for $-(\beta-\zeta G)$. Thus, if the initial condition, $x_{0}$, is in $\mathcal{B}_{0.31}(0)$, we are guaranteed decay to zero at an exponential rate. However, we shall show through numerical examples that the region of attraction for $x=0$ is much larger.

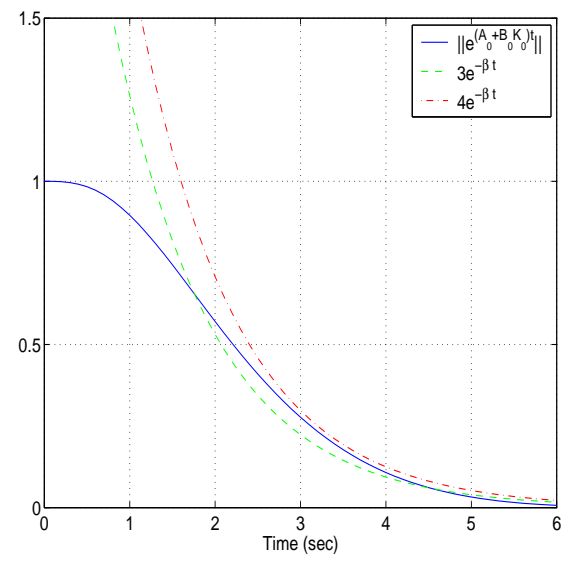

a

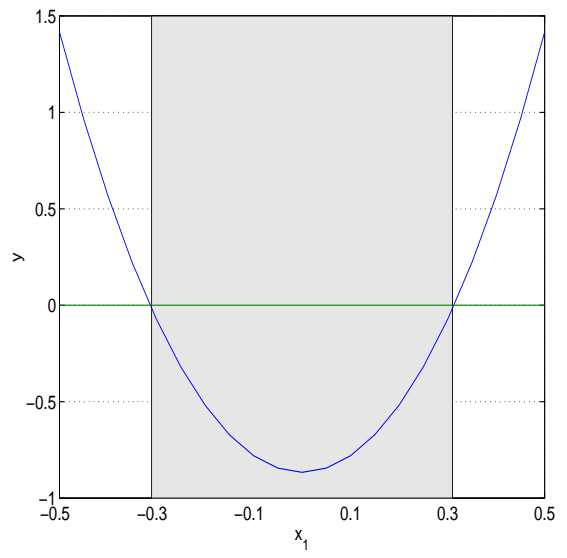

(b)

Figure 1: (a) Different values for $G$ in $G e^{-\beta t}$ and (b) plot of $y(28)$ vs. $x_{1}, y$ being negative (shaded) indicates region of attraction.

We first consider the system behavior when the initial condition is $x_{0}=(1,-1)^{T}$. Figure 2 (a) depicts the state dynamics of the controlled system while Figure 2(b) is the norm of the state dynamics. Not only do the dynamics exhibit decay to zero, but the graph of the norm exhibits exponential decay. Figure 2(c) is the control and Figure 2(d) is the value of the cost functional integrand over time.

The second initial condition that we consider is $x_{0}=(5,0)^{T}$. We find that, despite this initial condition being farther from the origin, the system also converges to zero asymptotically. Figures $3(\mathrm{a}), 3(\mathrm{~b}), 3(\mathrm{c})$, and $3(\mathrm{~d})$ correspond to the state, the norm of the state, the control and the cost functional integrand for the system with this initial condition. The control effort required to stabilize the system is large relative to that of the previous initial condition. In fact, the cost for the entire time interval is $J=4023.3$, whereas the cost for the first initial condition is 0.6346 . 


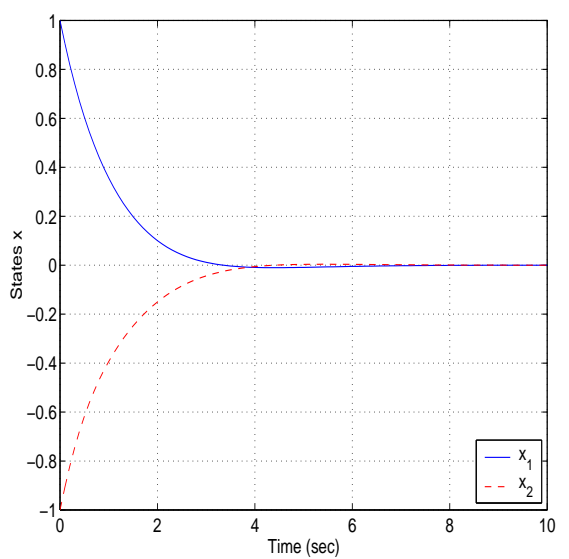

(a)

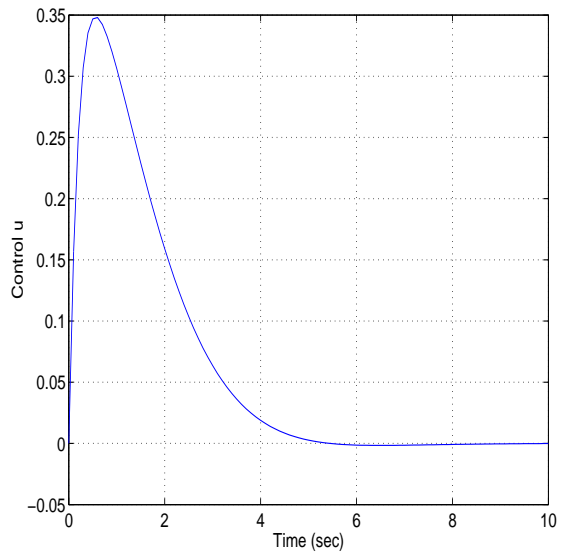

(c)

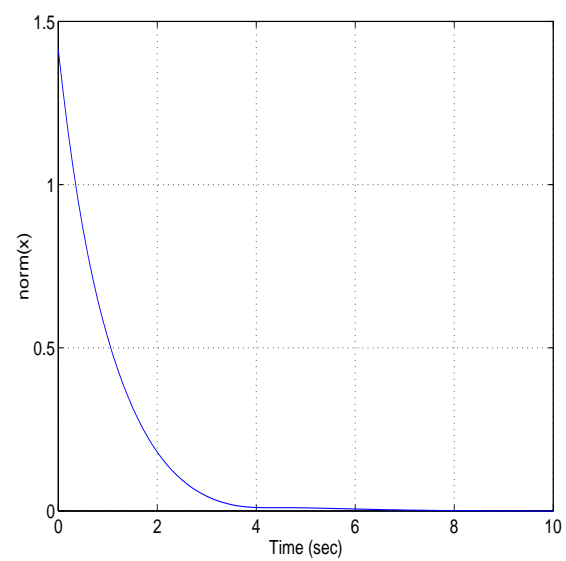

(b)

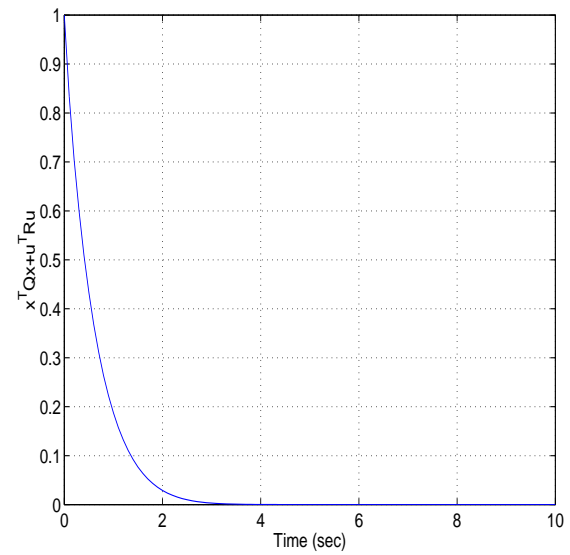

(d)

Figure 2: Full state feedback with exact SDRE solution, (a) closed loop state dynamics, (b) norm of the state dynamics, (c) SDRE formulated control $u$, and (d) cost functional integrand over the time span $0 \leq t \leq 10$ with the initial condition $x_{0}=(1,-1)^{T}$. 


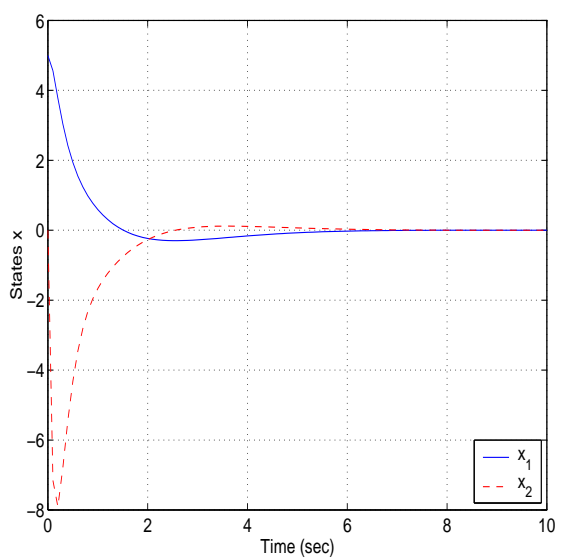

(a)

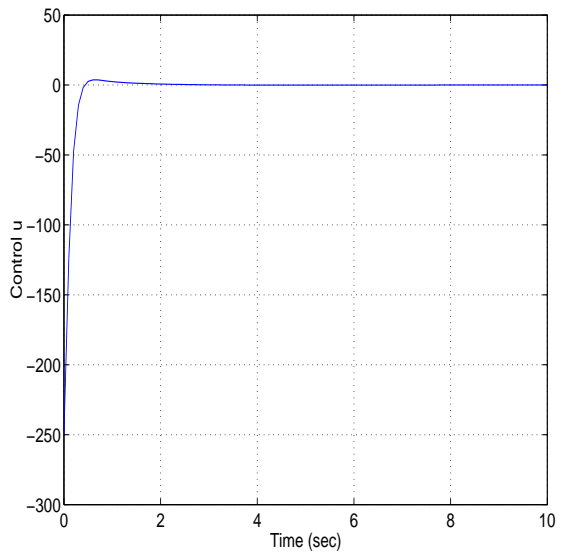

(c)

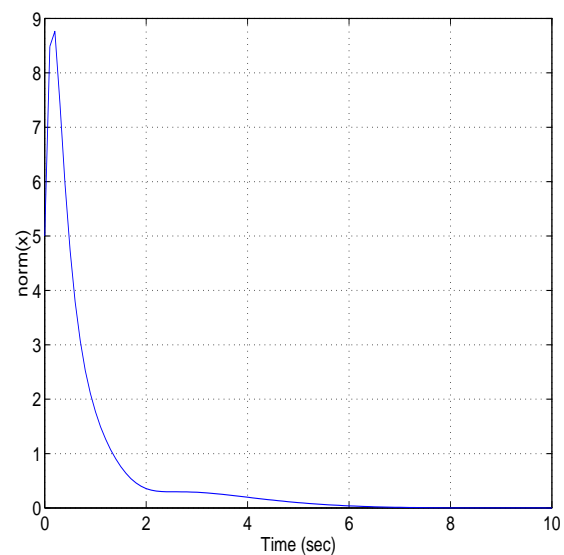

(b)

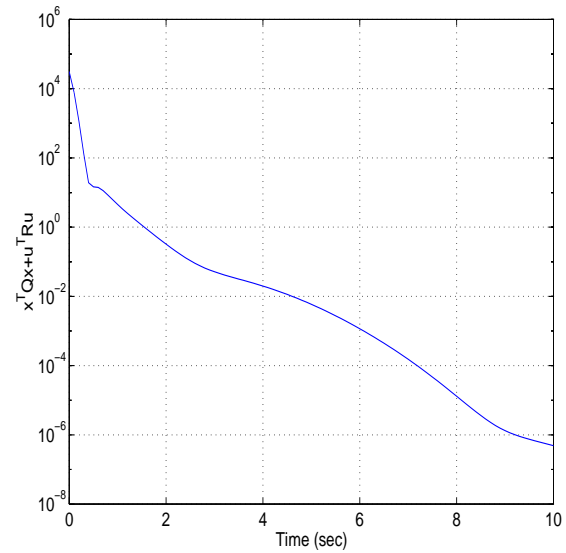

(d)

Figure 3: Full state feedback with exact SDRE solution, (a) closed loop state dynamics, (b) norm of the state dynamics, (c) SDRE formulated control $u$, and (d) cost functional integrand over the time span $0 \leq t \leq 10$ with the initial condition $x_{0}=(5,0)^{T}$. 


\section{$3 \quad$ Numerical Methods for Solving SDRE}

In Section 2.1, we presented an example for which the solution of the SDRE could be found analytically. In general one cannot find an exact solution analytically. Currently, one approach for solving the SDRE is via symbolic software packages such as Macsyma or Mathematica. However, once the dynamics of the system become complicated it is difficult to obtain a solution in this manner and it becomes necessary to approximate the solution. The method we describe in Section 3.1, referred to as the Taylor series method [7], works for systems with constant control coefficients $(B$ is not dependent on $x$ ). This uses the methodology of Taylor series approximations and is only effective locally. The interpolation method, presented in Section 3.2, can be used for more complex systems. This method involves varying the state over the domain of interest, solving for and storing the control $u(x)$, the SDRE solution $\Pi(x)$, or the SDRE gain $K(x)$ in a grid and interpolating over the stored solutions to approximate the control.

\subsection{Taylor Series Method}

The Taylor series method is used to synthesize controls for systems of the form

$$
\dot{x}=A(x) x+B u,
$$

where the system has the same characteristics as (2) except that $B$ is constant. Since the control coefficients are constant, the SDRE has the form

$$
\Pi(x) A(x)+A^{T}(x) \Pi(x)-\Pi(x) B R^{-1} B^{T} \Pi(x)+Q=0,
$$

where $Q$ and $R$ are the matrices from the cost functional (1).

We rewrite the matrix $A(x)$ as a sum of a constant matrix $\hat{A}$ and a state-dependent incremental matrix $\Delta A(x)$, where we choose $\hat{A}$ so that $(\hat{A}, B)$ is stabilizable. In order to form a series representation, the temporary variable $\epsilon$ (to be set to one upon completion of the series derivation) is introduced so that

$$
A(x)=\hat{A}+\epsilon \Delta A(x) .
$$

We also represent the solution of the SDRE as the Taylor series

$$
\begin{aligned}
\Pi(x, \epsilon) & =\left.\Pi(x)\right|_{\epsilon=0}+\left.\frac{\partial \Pi(x)}{\partial \epsilon}\right|_{\epsilon=0} \epsilon+\left.\frac{\partial^{2} \Pi(x)}{\partial \epsilon^{2}}\right|_{\epsilon=0} \frac{\epsilon^{2}}{2}+\ldots \\
& =\sum_{n=0}^{\infty} \epsilon^{n} L_{n}(x)
\end{aligned}
$$

where each $L_{n}$ matrix is symmetric as a result of the symmetry of $\Pi(x)$.

Substitution of these representations for $A(x)$ and $\Pi(x)$ into (29) yields

$$
\begin{aligned}
\left(\sum_{n=0}^{\infty} \epsilon^{n} L_{n}(x)\right)(\hat{A} & +\epsilon \Delta A(x))+(\hat{A}+\epsilon \Delta A(x))^{T}\left(\sum_{n=0}^{\infty} \epsilon^{n} L_{n}(x)\right) \\
& -\left(\sum_{n=0}^{\infty} \epsilon^{n} L_{n}(x)\right) B R^{-1} B^{T}\left(\sum_{n=0}^{\infty} \epsilon^{n} L_{n}(x)\right)+Q=0 .
\end{aligned}
$$


Grouping like powers of $\epsilon$ and setting the coefficients to zero, we obtain an iterative method for finding the $L_{n}$ matrices. This iteration scheme is

$$
\begin{aligned}
L_{0} \hat{A}+\hat{A}^{T} L_{0}-L_{0} B R^{-1} B^{T} L_{0}+Q & =0, \\
L_{1}\left(\hat{A}-B R^{-1} B^{T} L_{0}\right)+\left(\hat{A}^{T}-B R^{-1} B^{T} L_{0}\right) L_{1}+L_{0} \Delta A+\Delta A L_{0} & =0, \\
L_{n}\left(\hat{A}-B R^{-1} B^{T} L_{0}\right)+\left(\hat{A}^{T}-B^{T} R^{-1} B L_{0}\right) L_{n}+L_{n-1} \Delta A & \\
+\Delta A^{T} L_{n-1}-\sum_{k=1}^{n-1} L_{k} B R^{-1} B^{T} L_{n-k} &
\end{aligned}
$$

We note that equation (30) is the ARE corresponding to $(\hat{A}, B)$ while equations $(31)$ and $(32)$ are state-dependent Lyapunov equations. This algorithm converges locally to the solution of the SDRE under the assumptions that $A(x)$ and $B(x)$ are continuous [32]. This system of equations simplifies even further if $\Delta A(x)=g(x) \Delta A_{C}$, where $\Delta A_{c}$ is a constant matrix. By defining

$$
L_{n}(x)=(g(x))^{n}\left(L_{n}\right)_{C},
$$

with $\left(L_{n}\right)_{C}$ a constant matrix, we obtain the simplified iterative scheme

$$
\begin{aligned}
L_{0} \hat{A}+\hat{A}^{T} L_{0}-L_{0} B R^{-1} B^{T} L_{0}+Q & =0 \\
\left(L_{1}\right)_{C}\left(\hat{A}-B R^{-1} B^{T} L_{0}\right)+\left(\hat{A}^{T}-B R^{-1} B^{T} L_{0}\right)\left(L_{1}\right)_{C}+L_{0} \Delta A_{C}+\Delta A_{C} L_{0} & =0 \\
\left(L_{n}\right)_{C}\left(\hat{A}-B R^{-1} B^{T} L_{0}\right)+\left(\hat{A}^{T}-B^{T} R^{-1} B L_{0}\right)\left(L_{n}\right)_{C}+\left(L_{n-1}\right)_{C} \Delta A_{C} & =0 . \\
+\Delta A_{C}^{T}\left(L_{n-1}\right)_{C}-\sum_{k=1}^{n-1}\left(L_{k}\right)_{C} B R^{-1} B^{T}\left(L_{n-k}\right)_{C} &
\end{aligned}
$$

Therefore, when $A(x)$ is of this type, we can approximate $\Pi(x)$ using constant matrices calculated offline by solving one constant ARE and a series of constant Lyapunov equations. The subsequent control (an approximation of (14)) is given by

$$
u^{N}(x)=-R^{-1} B^{T}\left(\sum_{n=0}^{N}\left((g(x))^{n}\left(L_{n}\right)_{C}\right)\right) x,
$$

where $N$ is the number of members of the series computed offline.

If solving the Lyapunov equations in real-time is not feasible and $\Delta A(x)$ does not take on the form described above, only $L_{0}$ and $L_{1}$ can be computed numerically offline (without the use of symbolic solutions to the state-dependent Lyapunov equations). To achieve this, we rewrite

$$
A(x)=\hat{A}+\sum_{j=1}^{m} f_{j}(x)\left(\Delta A_{j}\right)_{C},
$$

where $f_{j}(x)$ are the distinct nonlinear terms of $A(x)$ and $\left(\Delta A_{j}\right)_{C}$ are the corresponding constant matrix coefficients. If we assume $L_{1}$ can be written as

$$
L_{1}=\sum_{j=1}^{m} f_{j}(x)\left(L_{1}^{j}\right)_{C},
$$

group the $f_{j}(x)$ terms together for each $j$, and set the coefficients to zero, we can approximate the solution to the SDRE by solving

$$
\begin{gathered}
L_{0} \hat{A}+\hat{A}^{T} L_{0}-L_{0} B R^{-1} B^{T} L_{0}+Q=0 \\
\left(L_{1}^{j}\right)_{C}\left(\hat{A}-B R^{-1} B^{T} L_{0}\right)+\left(\hat{A}^{T}-B R^{-1} B^{T} L_{0}\right)\left(L_{1}^{j}\right)_{C} \\
\quad+L_{0}\left(\Delta A_{C}^{j}\right)+\left(\Delta A_{C}^{j}\right) L_{0}=0, j=1, \ldots, m .
\end{gathered}
$$


The corresponding approximate control $\left(u^{1}\right.$ denoting that we use one term, in addition to $L_{0}$, of the Taylor series) is given by

$$
u^{1}(x)=-R^{-1} B^{T}\left(L_{0}+\sum_{j=1}^{m} L_{1}^{j} f_{j}(x)\right) x .
$$

\subsection{Interpolation Method}

The interpolation method, alluded to in [21], involves formulating the SDRE control $(u(x))$, SDRE solution $(\Pi(x))$, or even the SDRE gain matrix $(K(x))$ at a number of states in the domain of consideration and creating a grid to interpolate over as the states vary. We are able to construct an interpolation grid because the solutions to the SDRE continuously depend on the state. This technique is similar in spirit to gain scheduling and an optimal control two point boundary value problem described in [16]. We now proceed to develop the method mathematically.

\subsubsection{Interpolate the Control}

Let $\Omega_{0}$ denote the set from which the initial conditions are chosen. We assume that the state space, $\Omega$, is such that the state trajectory for any initial condition, $x_{0} \in \Omega_{0}$, is contained entirely in $\Omega$. If $n$ is the dimension of the state, we create a finite mesh, $D$, that varies each $x_{i}, i=1, \ldots, n$ over $\Omega$. At each $\hat{x} \in D$, solve and store the resulting control, $u_{\hat{x}}$. Thus, we have effectively created an interpolation grid that can be used to estimate the control for any point in $\Omega$. In other words, the control can be approximated by

$$
u(x)=\operatorname{interp}\left\{u_{\hat{x}}\right\}
$$

where interp $\{*\}$ represents any type of interpolation (1-d, 2-d, cubic, linear, etc.).

\subsubsection{Interpolate over $\Pi(x)$}

In SDC form, we find that the number of state variables on which $A(x)$ and $B(x)$ depend is often less than the dimension of the system. Let $\Omega_{0}$ and $\Omega$ be defined as above. If $n$ is the dimension of the state, we denote the $r \leq n$ states present in $A(x)$ and $B(x)$ as $\Xi=\left\{x_{i_{1}}, \ldots, x_{i_{r}}\right\}$. If we create a mesh, $D$, that varies over each $x_{i_{j}} \in \Xi$ over the bounds imposed by $\Omega$, solve and store the resulting constant SDRE for each $x \in D$, we have effectively created an interpolation grid for each element of $\Pi(x)$. Thus, to estimate the solution to the SDRE for any point in $\Omega$, we can interpolate over the elements of the grid. Thus, we can approximate the control with

$$
u(x)=-R^{-1} B^{T}(x)\left[\begin{array}{ccc}
\operatorname{interp}\left\{\pi_{11}(x)\right\} & \ldots & \operatorname{interp}\left\{\pi_{1 n}(x)\right\} \\
\vdots & \ddots & \vdots \\
\operatorname{interp}\left\{\pi_{n 1}(x)\right\} & \ldots & \operatorname{interp}\left\{\pi_{n n}(x)\right\}
\end{array}\right] x
$$

where $\operatorname{interp}\{*\}$ is defined as above. Since $\Pi(x)$ is symmetric, we have $\operatorname{interp}\left\{\pi_{i j}\right\}=\operatorname{interp}\left\{\pi_{j i}\right\}$ and the number of $r$-degree interpolations is then $n(n+1) / 2$.

Remark 3.1. We make the following comments regarding the interpolation method:

(i) The number of points $(M)$ used for the mesh should be sufficiently large so that the closed loop system is approximated accurately (otherwise, there might be instability). 
(ii) Interpolation over $\Pi(x)$ is advantageous when the number of states, $r$, in $\Xi$ is less than $n$. The degree of the interpolation is less for each element of $\Pi(x)$ and the elements not present in $\Xi$ do not have bounds invoked by the mesh.

(iii) Interpolation over $u(x)$ is advantageous when the number of states, $r$, in $\Xi$ is equal to $n$ since both meshes will impose bounds on all states and the interpolation is of the same complexity.

(iv) If $n m \leq n(n+1) / 2$, where $m$ is the dimension of the control, then the interpolation method can be used with the gain matrix, $K(x)$ to decrease the number of interpolations needed.

(iv) Not only can the interpolation method be used for nonconstant $B(x)$, but it can also be used in more complex SDRE formulations that have nonconstant weighting matrices, $Q(x)$ and $R(x)$, in the cost functional.

\subsection{Example 1 Revisited: Numerical Approximations}

In Section 2.1 the exact solution to (26), (27) was used for the control of the system. We now use the numerical methods introduced in the previous section to approximate the solution to the SDRE, synthesize controls based on the approximations, and compare the results to the exact solution. To generate the Taylor series, we use the same representations as in Section 2 to split $A(x)$ into a constant and state-dependent part. Therefore, we have

$$
\hat{A}=\left[\begin{array}{ll}
0 & 1 \\
0 & 0
\end{array}\right], \quad \Delta A(x)=\left[\begin{array}{ll}
0 & 0 \\
1 & 0
\end{array}\right] x_{1}^{2} .
$$

Since $\Delta A(x)=A_{C} g(x)$, we can generate as many terms of the Taylor series offline as desired. For the interpolation methods (both $u(x)$ and $\Pi(x)$ ), we create four grids INTu1, INTu2, INTPI1, and INTPI2 generated by two different meshes; one that uses a constant increment and another that focuses more attention (a finer mesh) closer to zero. Specifically, the first mesh $D_{1}$ (corresponding to INTu1 and INTPI1), is varied from [-5,5] in steps of 0.5 whereas the second mesh, $D_{2}$ (corresponding to INTu2 and INTPI2), takes the values $x \in\{-5,-3.5,-2.5,2.5,3.5,5\} \cup\{-1: 0.25: 1\}$, where $\{a: b: c\}$ indicates incrementing from $a$ to $c$ in steps of $b$. For interpolating the control, $u(x)$, we vary both $x_{1}$ and $x_{2}$ over the values in each mesh and for $\Pi(x)$ we only vary $x_{1}$ since $x_{2}$ does not appear in $A(x)$.

As mentioned in Section 3.1, there are limitations when using the Taylor series method for approximating the SDRE outside of a small interval about the origin. For instance, in [7], the approximate SDRE control fails for the system (26), (27) with initial conditions $x_{0}=(2,0)^{T}$ and $x_{0}=(4,0)^{T}$. This occurs when five terms of the Taylor series method are used to approximate $\Pi(x)$. The instability can be examined more completely if we consider the maximum real part of the eigenvalues of the closed loop matrix $A(x)-B K(x)$ over an interval of $x_{1}$. If the maximum real part of these eigenvalues crosses the y-axis, the estimation for $\Pi(x)$ is not only inaccurate but also causes the system to become unstable. Figure 4(a) depicts the maximum real part of the eigenvalues of $A(x)-B K(x)$ over $x_{1} \in\{-5: 0.2: 5\}$ when Taylor series of different lengths were used to synthesize the control. It can be seen that the real part of the eigenvalue for the Taylor series approximations are close to the exact solution locally. We see that if the 2-term and 8-term expansions are used for the approximations to $\Pi(x)$, then the closed loop system will have eigenvalues with negative real parts. The other expansions displayed in the plot produce unstable behavior outside a small neighborhood of the origin. In fact, the expansion with five terms has eigenvalues with negative real parts in the smallest interval of the six presented. Therefore, it is 


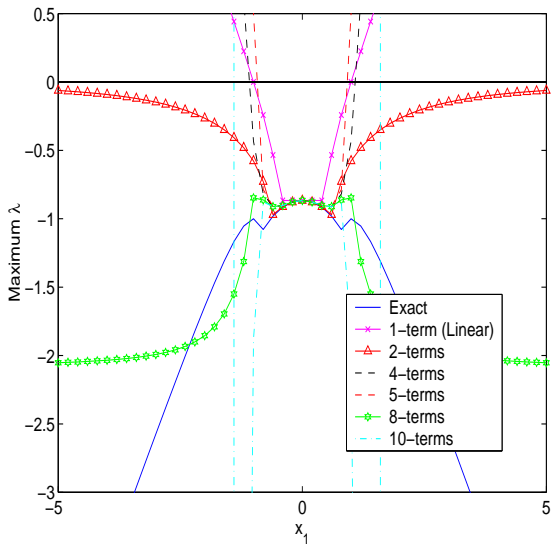

(a)

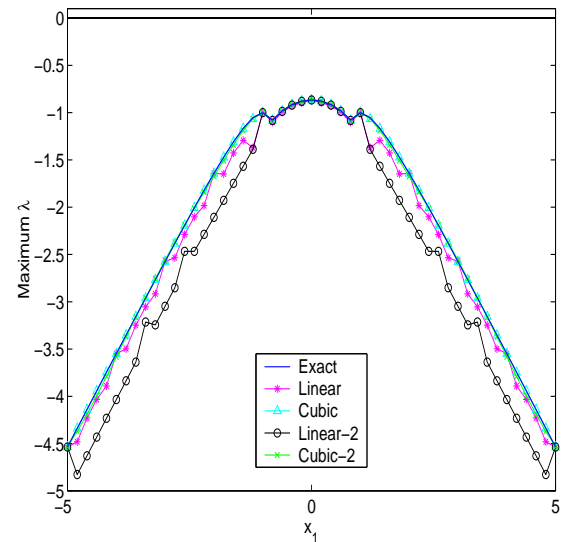

(b)

Figure 4: Maximum real part of the eigenvalues of the closed loop control matrix for (a) Taylor series method and (b) $\Pi(x)$ interpolation grids INTPI1 and INTPI2.

not a surprise that there were difficulties using the control for the system with the initial condition $(2,0)^{T}$ and $(4,0)^{T}$. We see from this example that using more terms in the Taylor series will not necessarily result in more accuracy. For instance, we observe that the use of the 8-term expansion in control formulation results in a closed loop system that remains stable for the entire interval whereas the 10-term series results in an unstable closed loop system outside of a small region of the origin. For the sake of comparison, we provide the plot for the maximum real part of the eigenvalues of the closed loop control for the interpolation method (over $\Pi(x)$ ) in Figure 4(b). Here, the maximum real part of the eigenvalues is close to exact for both cubic and linear interpolation for the entire interval.

Next, to study the accuracy of all of the numerical approaches, we use the approximate feedback controls to stabilize the system from a number of initial conditions. Since the 2-term and 8-term Taylor series produce the only stable closed loop systems, we present the results for the controls generated with these two series. We use initial conditions close to the origin and relatively far from the origin, so as to compare the Taylor series method to the interpolation method in both situations. These initial conditions are $(1,0)^{T}$ and $(3,3)^{T}$, respectively.

Table 3.3 lists the floating point operation count (FLOPS) needed at each time step to compute the control, the 2-norm of the difference between the numerical method and the exact solution, and the value of (26) for both initial conditions. Since INTu2 does not have a uniform mesh, it requires more FLOPS to use interpolation. As can be seen in the table, one 2-d cubic interpolation for $u(x)$ is more costly than the three 1-d cubic interpolations for the $\Pi(x)$ grid and the control formulated using the $\Pi(x)$ grid is also the most accurate for both types of interpolation. Figures 5(a), 5(b), $5(\mathrm{c})$, and 5(d) represent the states $x_{1}$ and $x_{2}$, the control, and the cost functional integrand for this example with the initial condition $x_{0}=(1,0)^{T}$. The solutions presented graphically are the 2-term Taylor series and control interpolation over INTu1 and INTu2 using linear interpolation. The 2-term Taylor series is presented because it has lower cost and is the more accurate of the two stable Taylor series representations. The specific interpolation methods are displayed because they are the worst performing of the interpolation method and still relatively accurate and low cost compared to the Taylor series approximation. The second interpolation grid (INTu2), as mentioned, was created for better performance near the origin. This is reflected in the figures since the control 


\begin{tabular}{|l|c|c|c|c|c|}
\hline Method & FLOPS & D1 & C1 & D2 & C2 \\
\hline Exact & 22 & 0.000 & 1.967 & 0.000 & $7.677 \times 10^{2}$ \\
\hline 2-term TS & 34 & $2.933 \times 10^{-1}$ & 1.833 & 9.872 & $1.231 \times 10^{3}$ \\
\hline 8-term TS & 100 & $1.105 \times 10^{-1}$ & 2.246 & 4.722 & $7.717 \times 10^{14}$ \\
\hline INTu1: Linear & 39 & $2.466 \times 10^{-1}$ & 2.009 & $3.637 \times 10^{-1}$ & $7.688 \times 10^{2}$ \\
\hline INTu1: Cubic & 475 & $3.623 \times 10^{-2}$ & 1.964 & $2.492 \times 10^{-2}$ & $7.676 \times 10^{2}$ \\
\hline INTPI1: Linear & 69 & $9.062 \times 10^{-2}$ & 1.980 & $1.047 \times 10^{-1}$ & $7.682 \times 10^{2}$ \\
\hline INTPI1: Cubic & 162 & $5.334 \times 10^{-3}$ & 1.967 & $5.909 \times 10^{-3}$ & $7.677 \times 10^{2}$ \\
\hline INTu2: Linear & 121 & $6.564 \times 10^{-2}$ & 1.976 & 2.112 & $8.401 \times 10^{2}$ \\
\hline INTu2: Cubic & 6935 & $1.556 \times 10^{-3}$ & 1.966 & $3.541 \times 10^{-2}$ & $7.681 \times 10^{2}$ \\
\hline INTPI2: Linear & 138 & $2.420 \times 10^{-2}$ & 1.970 & $7.587 \times 10^{-1}$ & $7.914 \times 10^{2}$ \\
\hline INTPI2: Cubic & 2280 & $1.011 \times 10^{-4}$ & 1.967 & $1.628 \times 10^{-2}$ & $7.683 \times 10^{2}$ \\
\hline
\end{tabular}

Table 1: Comparison of Numerical Methods, FLOPS - the floating point operations needed to compute the control for one time step, the 2-norm of the difference of the approximate state trajectory and the exact trajectory for (D1) $x_{0}=(1,0)^{T}$ and (D2) $x_{0}=(3,3)^{T}$, and the value of the cost functional integrand for $(\mathrm{C} 1) x_{0}=(1,0)^{T}$ and $(\mathrm{C} 2) x_{0}=(3,3)^{T}$.

and subsequent dynamics are closer to those generated by the exact solution than those generated by the uniform grid (INTu1). The Taylor series approach also produces a control that is similar to the exact for this relatively local initial condition.

The second initial condition, $(3,3)^{T}$ is further from the origin. However, all of the methods produce stabilizing controls. Figures $6(\mathrm{a}), 6(\mathrm{~b}), 6(\mathrm{c})$, and $6(\mathrm{~d})$ represent the states $x_{1}$ and $x_{2}$, the control, and the cost integrand for this initial condition. The Taylor series method depicted (as well as the 8-term, not depicted) produces an approximate feedback control that is far from accurate. The interpolation method produces relatively accurate controls without much computational effort.

\subsection{D Example}

To illustrate a case when interpolating over $\Pi(x)$ or $K(x)$ is more beneficial, we consider the following example from [31]. The system is

$$
\dot{x}=\left[\begin{array}{c}
x_{2} \\
x_{3} \\
x_{4}^{3} \\
x_{5}+x_{4}^{3}-x_{1}^{2} \\
0
\end{array}\right]+\left[\begin{array}{ll}
0 & 0 \\
0 & 0 \\
1 & 0 \\
0 & 0 \\
0 & 1
\end{array}\right] u .
$$

Associated with these dynamics, we choose the quadratic cost functional weighting matrices in (1) to be $Q=10 I_{5 \times 5}$ and $R=0.1 I_{2 \times 2}$.

Here, we use an obvious SDC parameterization

$$
A(x)=\left[\begin{array}{ccccc}
0 & 1 & 0 & 0 & 0 \\
0 & 0 & 1 & 0 & 0 \\
0 & 0 & 0 & x_{4}^{2} & 0 \\
-x_{1} & 0 & 0 & x_{4}^{2} & 1 \\
0 & 0 & 0 & 0 & 0
\end{array}\right]
$$




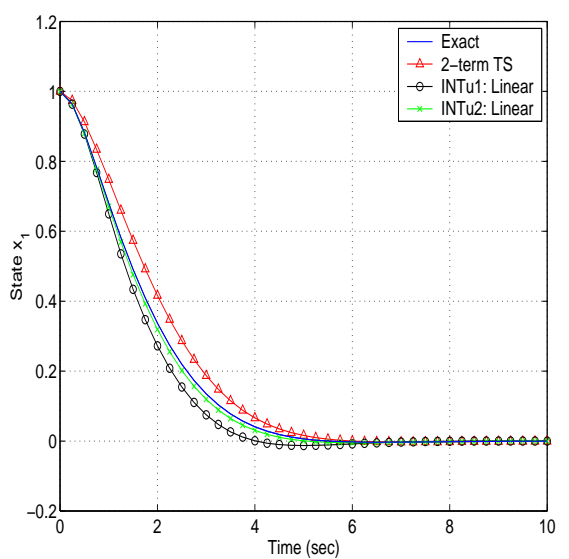

(a)

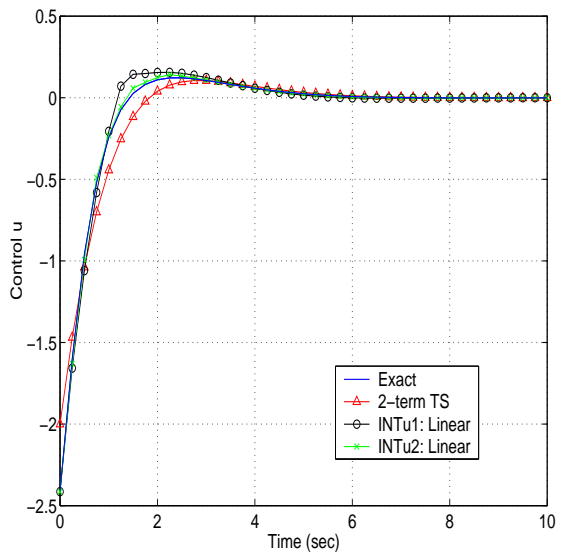

(c)

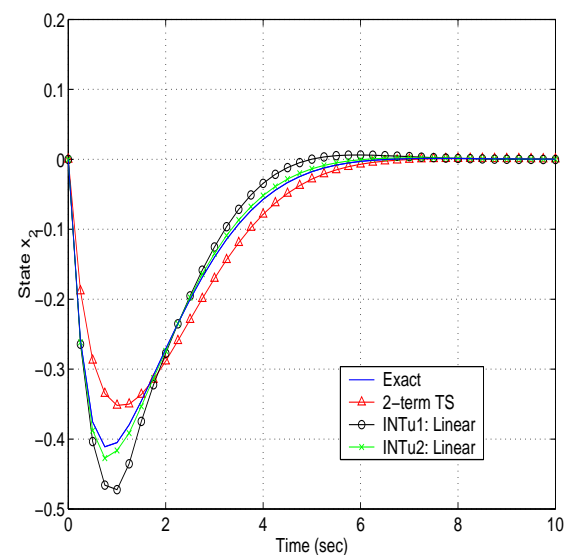

(b)

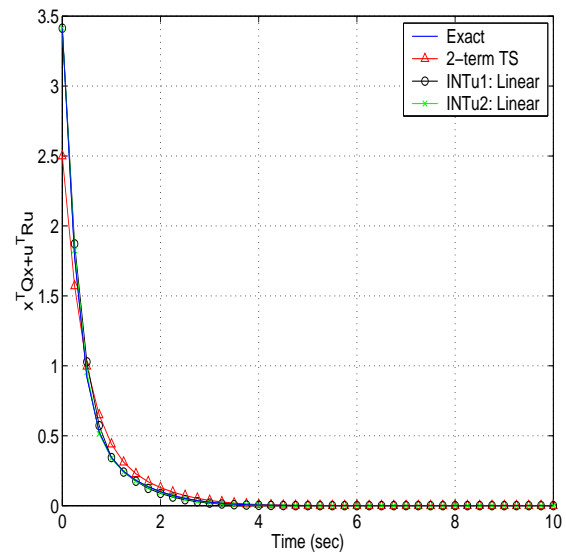

(d)

Figure 5: Comparison of Taylor series and interpolation methods for 2D example, closed loop state dynamics for (a) $x_{1}$ and (b) $x_{2}$, (c) control $u$, and (d) cost functional integrand over the time span $0 \leq t \leq 10$ with $x_{0}=(1,0)^{T}$. 


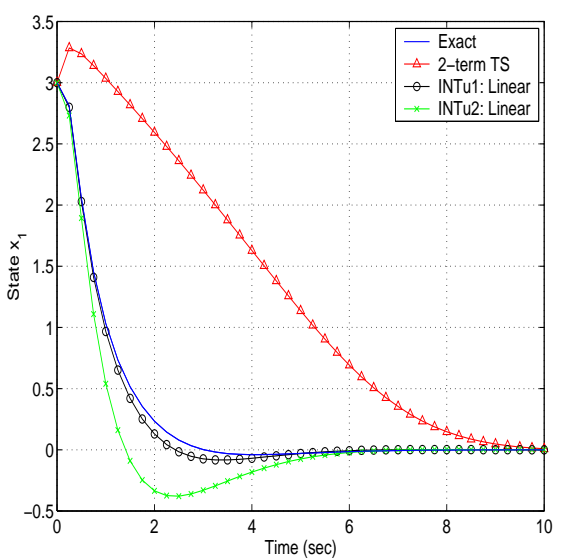

(a)

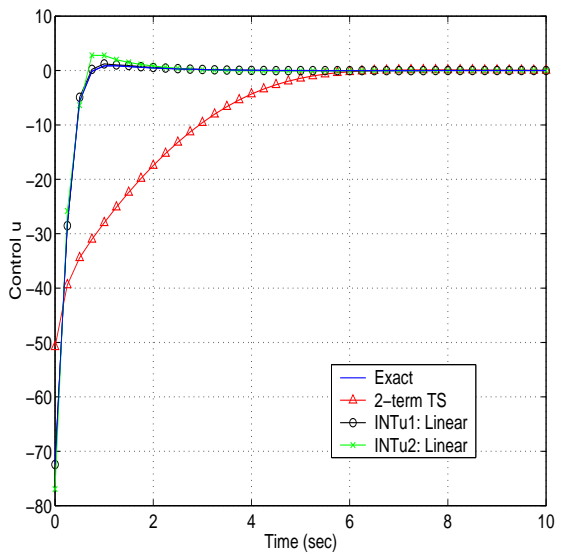

(c)

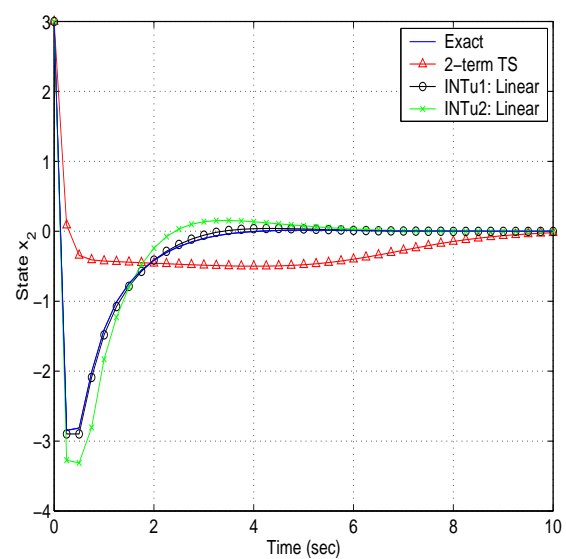

(b)

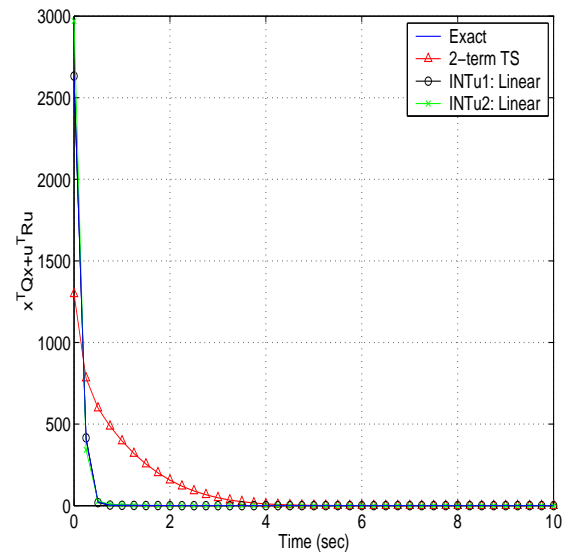

(d)

Figure 6: Comparison of Taylor series and interpolation methods for 2D example, closed loop state dynamics for (a) $x_{1}$ and (b) $x_{2}$, (c) control $u$, and (d) cost functional integrand from $0 \leq t \leq 10$ with $x_{0}=(3,3)^{T}$. 


\begin{tabular}{|l|c|}
\hline Method & FLOPS \\
\hline INTPI: Linear & 2028 \\
\hline INTK: Linear & 1292 \\
\hline INTu: Linear & 2202 \\
\hline
\end{tabular}

Table 2: Comparison of interpolation methods for 5D example.

The first $n=5$ columns of the state-dependent controllability matrix are

$$
M(x)=\left[\begin{array}{lllll}
0 & 0 & 0 & 0 & 1 \\
0 & 0 & 1 & 0 & 0 \\
1 & 0 & 0 & 0 & 0 \\
0 & 0 & 0 & 1 & 0 \\
0 & 1 & 0 & 0 & 0
\end{array}\right]
$$

which has rank $n$. There are only two states present in the $A(x)$ matrix so that $\Xi=\left\{x_{1}, x_{4}\right\}$. Hence, when creating the $\Pi(x)$ and $K(x)$ grids (INTPI and INTK, respectively), we need only vary over those two particular states. Both of the grids use the mesh

$$
D_{14}=\left\{x_{1}=-2: 0.5: 2\right\} \times\left\{x_{4}=-2: 0.2: 2\right\} .
$$

When creating the grid for interpolation over $u$, we must create a grid in five dimensions. This task is accomplished by crossing the $D_{14}$ mesh with $D_{j}=\left\{x_{j}=-2: 1: 2\right\}, j=2,3,5$. Therefore, the offline calculations take more time and computational effort to produce the grid. The control is formulated at each time step by either two 5D linear interpolations for $u$, ten 2D linear interpolations for $K(x)$ or fifteen 2D linear interpolations for $\Pi(x)$. Because of the complexity of $5 \mathrm{D}$ interpolations, interpolation over the $u$ grid is not only less accurate but more computationally taxing. Figures $7(\mathrm{a})-(\mathrm{d})$ represent states $x_{2}, x_{4}, x_{5}$, and control $u_{2}$ respectively. The number of FLOPS that it took to formulate the control at each time step for the different methods is given in Table 2. Here, interpolation over the gain matrix $K(x)$ is clearly the most accurate and least computationally taxing. Note that in each of the following two examples the "exact" solution is obtained by solving the SDRE evaluated at the state (so that it is an ARE) at each time increment of the integration.

\subsection{D Example}

The last example we present is referred to as the toy example [13]. We present this example because it exhibits complexities not yet encountered in the previous examples. First, it has nonconstant weighting matrices for the state. Specifically, we seek to minimize the cost functional

$$
J\left(x_{0}, u\right)=\int_{0}^{\infty} x^{T} Q(x) x+u^{T} R u d t
$$

where

$$
Q(x)=0.01\left[\begin{array}{ccc}
0 & 0 & 0 \\
0 & e^{x_{1}} & 1 \\
0 & 1 & e^{-x_{1}}
\end{array}\right]
$$

with respect to the state dynamics

$$
\begin{aligned}
& \dot{x}_{1}=f_{1}(x) \\
& \dot{x}_{2}=e^{-x_{1}} x_{3}+e^{-x_{1} / 2} u, \\
& \dot{x}_{3}=-e^{x_{1}} x_{2}+e^{x_{1} / 2} u
\end{aligned}
$$




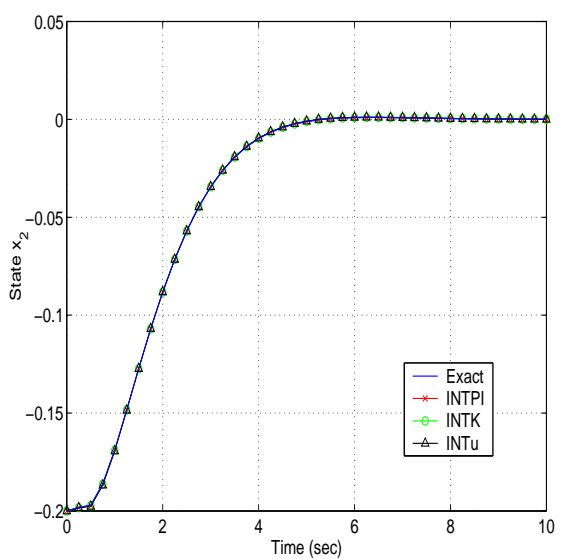

(a)

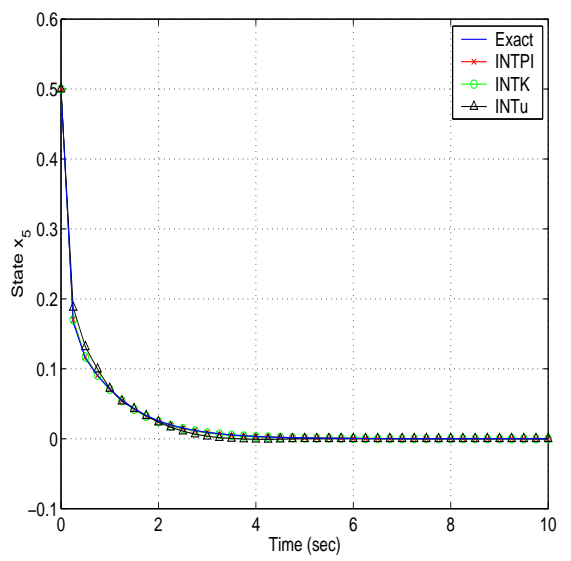

(c)

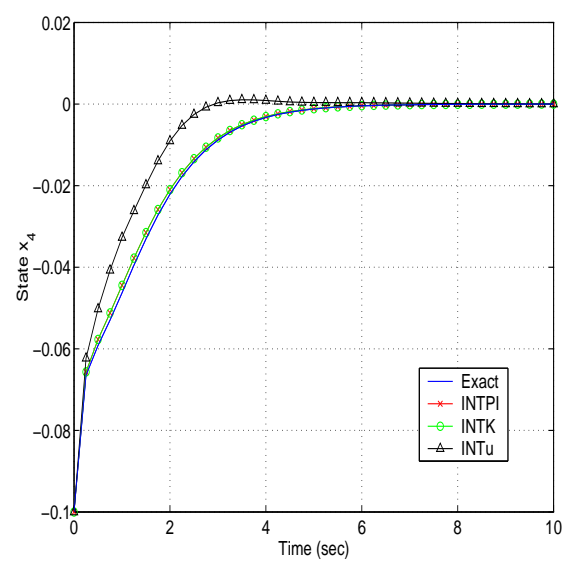

(b)

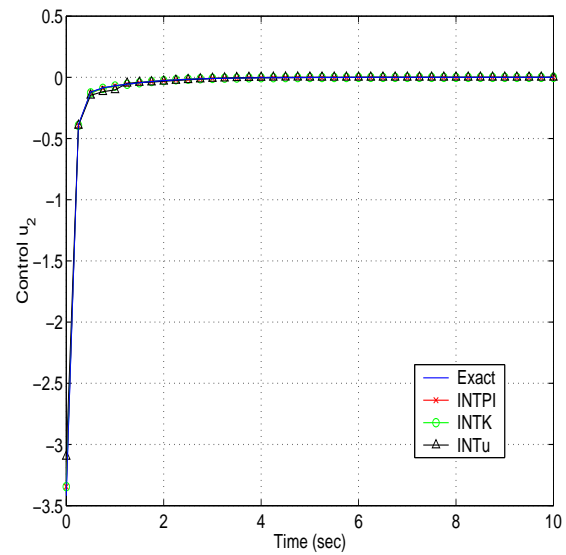

(d)

Figure 7: Comparison of interpolation methods for 5D example, closed loop state dynamics for (a) $x_{2}$, (b) $x_{4}$, and (c) $x_{5}$, and (d) control $u_{2}$ over the time span $0 \leq t \leq 10$ with $x_{0}=(0.4,-0.2,0.1,-0.1,0.5)^{T}$. 


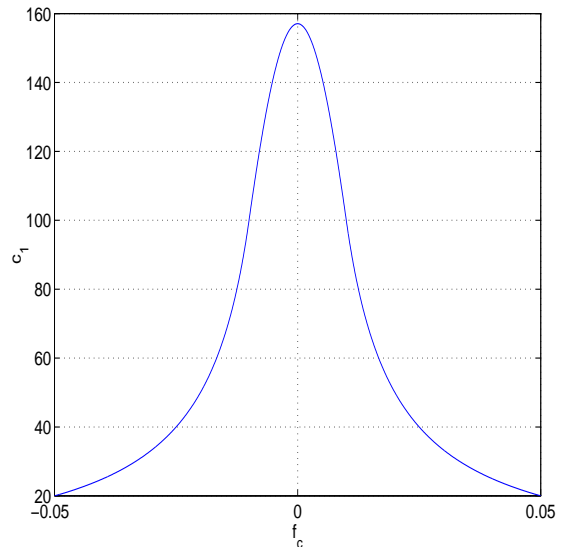

(a)

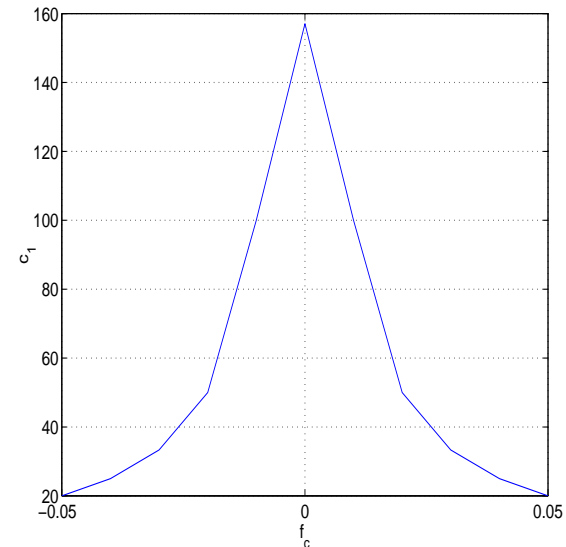

(b)

Figure 8: $c_{1}$ with $f_{c}$ step size (a) 0.0001 and (b) 0.01 .

where

$$
f_{1}(x)=\chi_{[-3,3]}\left(x_{1}\right) \operatorname{sgn}\left(e^{x_{1}} x_{2}^{2}-e^{-x_{1}} x_{3}^{2}\right) .
$$

Second, since the dynamics are not continuous we must use an approximate continuous parameterization to formulate the SDRE control. As detailed in [13], the $x_{1}$ dynamics used to formulate the control are approximated and stabilized using

$$
\dot{x}_{1} \approx \operatorname{satsin}\left(f_{c}\right)-x_{1},
$$

where

$$
\begin{gathered}
\operatorname{satsin}(\theta)=\left\{\begin{array}{ll}
\sin \left(\frac{\pi \theta}{2 \delta}\right) & \text { if }|\theta|<\delta \\
1 & \text { if } \theta \geq \delta \\
-1 & \text { if } \theta \leq-\delta
\end{array},\right. \\
f_{c}=e^{x_{1}} x_{2}^{2}-e^{-x_{1}} x_{3}^{2},
\end{gathered}
$$

and $\delta=0.001$. Then, defining

$$
c_{1}=\frac{\operatorname{satsin}\left(f_{c}\right)}{f_{c}}, \quad c_{2}=\frac{e^{x_{1}-1}}{x_{1}}, \text { and } \quad c_{3}=\frac{e^{-x_{1}-1}}{x_{1}},
$$

we can rewrite the dynamics used to formulate the control as

$$
\begin{aligned}
& \dot{x}_{1} \approx c_{1}\left(c_{2} x_{1}+1\right) x_{2}^{2}-c_{1}\left(c_{3} x_{1}+1\right) x_{3}^{2}-x_{1} \\
& \dot{x}_{2}=\left(c_{3} x_{1}+1\right) x_{3}+e^{-x_{1} / 2} u \\
& \dot{x}_{3}=-\left(c_{2} x_{1}+1\right) x_{2}+e^{x_{1} / 2}
\end{aligned}
$$

This introduces the last complexity that is of importance. The limits of $c_{1}, c_{2}$, and $c_{3}$ as $f_{c}$ and $x_{1}$ go to zero exist (and are $2 /(\delta \pi), 1$, and -1 respectively) and, hence, each are well behaved. However, as we increase the increment of $f_{c}$ from 0.0001 to 0.01 , it decreases the appearance of continuity in $c_{1}$ (see Figure 8). Therefore, the grid step size must be kept reasonably small to keep the closed loop system stable. This is also problematic for time integration, even using the exact solution. As can be seen in [13], there is some discontinuous behavior depicted in the plots of the control. In fact, we found that we had to increase the tolerance (of the Matlab routine ode15s) in 


\begin{tabular}{|l|c|}
\hline Method & FLOPS \\
\hline INTPI: Linear & 1413 \\
\hline INTK: Linear & 696 \\
\hline INTu: Linear & 246 \\
\hline
\end{tabular}

Table 3: Comparison of interpolation methods for 3D example.

order to integrate the system with the exact solution (the SDRE being solved at each time step) for some initial conditions.

By (4), we can parameterize these dynamics in many ways. In [13] (here we correct a mistake made therein), the terms with more than one multiple of the state are represented as

$$
\begin{aligned}
c_{1} c_{2} x_{1} x_{2}^{2} & =\alpha_{1}\left(c_{1} c_{2} x_{2}^{2}\right) x_{1}+\left(1-\alpha_{1}\right)\left(c_{1} c_{2} x_{1} x_{2}\right) x_{2}, \\
c_{1} c_{3} x_{1} x_{3}^{2} & =\alpha_{2}\left(c_{1} c_{3} x_{3}^{2}\right) x_{1}+\left(1-\alpha_{2}\right)\left(c_{1} c_{3} x_{1} x_{3}\right) x_{3}, \\
c_{3} x_{1} x_{3} & =\alpha_{3}\left(c_{3} x_{1}\right) x_{3}+\left(1-\alpha_{3}\right)\left(c_{3} x_{3}\right) x_{1}, \\
c_{2} x_{1} x_{2} & =\alpha_{4}\left(c_{2} x_{2}\right) x_{1}+\left(1-\alpha_{4}\right)\left(c_{2} x_{1}\right) x_{2} .
\end{aligned}
$$

We choose $\alpha_{1}=\alpha_{2}=0$ and $\alpha_{3}=\alpha_{4}=1$ resulting in the parameterization

$$
A(x)=\left[\begin{array}{ccc}
-1 & c_{1} c_{2} x_{1} x_{2}+c_{1} x_{2} & -c_{1} c_{3} x_{1} x_{3}-c_{1} x_{3} \\
c_{3} x_{3} & 0 & 1 \\
-c_{2} x_{2} & -1 & 0
\end{array}\right]
$$

and

$$
B(x)=\left[\begin{array}{c}
0 \\
e^{-x_{1} / 2} \\
e^{x_{1} / 2}
\end{array}\right] .
$$

The grids that we use result from varying $x_{1}, x_{2}$ and $x_{3}$ over the mesh $D=\{-3.5: 0.2: 3.5\}$. As depicted in Figure 9, interpolation over $\Pi(x), K(x)$, and $u(x)$ all stabilize the system. The FLOPS needed to compute the solution for each method are given in Table 3 . Since $u(x)$ only requires one interpolation, it is evident that interpolation over this grid is the most efficient method to approximate the control.

\section{Nonlinear Estimation}

We now extend the SDRE theory for state feedback to state estimation. This is of particular interest for nonlinear systems with state-dependent output coefficients. Specifically, for systems that can be put in the form

$$
\dot{x}=f(x)=A(x) x,
$$

where $x \in \Re^{n}$ and $f(x)$ is nonlinear with output given by

$$
y=C(x) x,
$$

where $C(x)$ is a continuous $m \times n$ matrix-valued function. From [14], we see that a system of the form

$$
\dot{x}_{e}=A\left(x_{e}\right) x_{e}+\Psi\left(x_{e},\left(C(x) x-C\left(x_{e}\right) x_{e}\right)\right)
$$

is a state estimator for this system if the error is asymptotically stable at the zero equilibrium. 


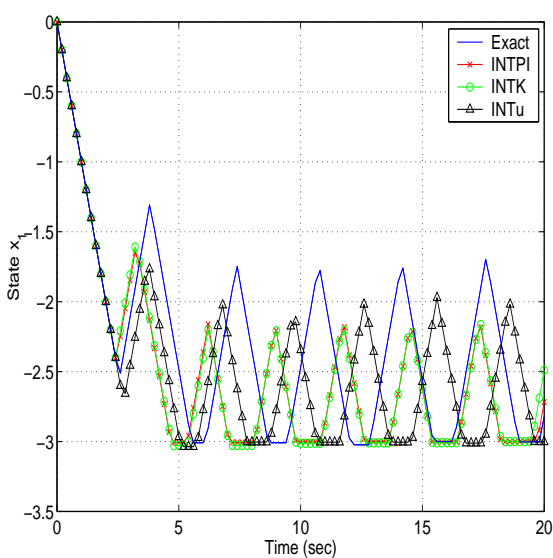

(a)

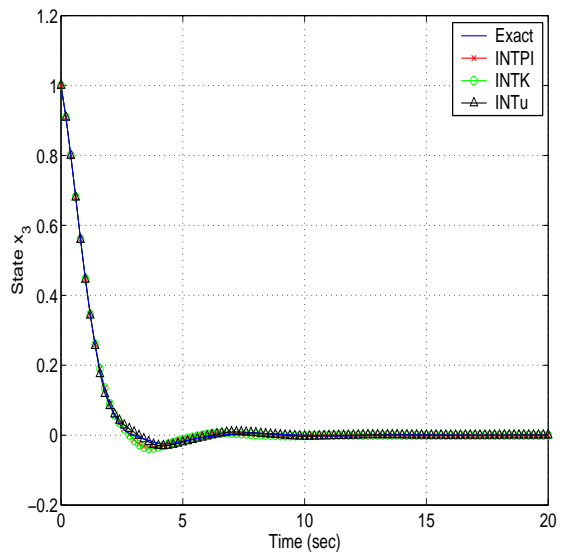

(c)

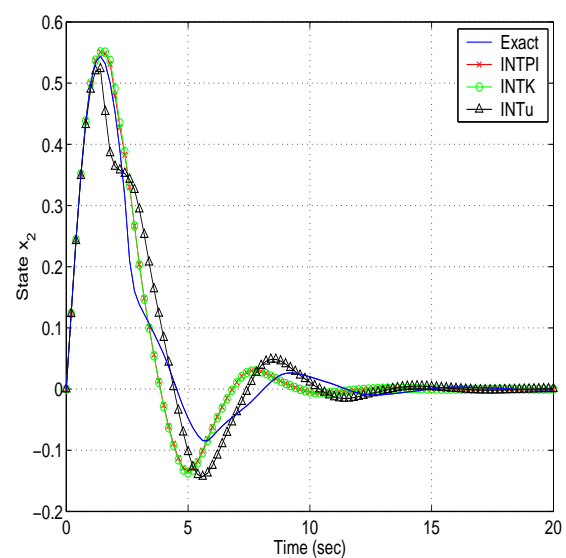

(b)

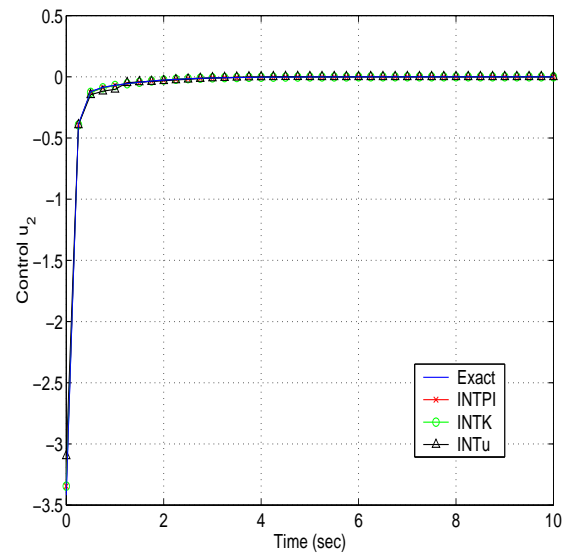

(d)

Figure 9: Comparison of interpolation methods for 3D example, closed loop state dynamics for (a) $x_{1}$, (b) $x_{2}$, and (c) $x_{3}$, and (d) control $u$ over the time span $0 \leq t \leq 20$ with $x_{0}=(0,2,3)^{T}$. 
We can now formulate the proposed nonlinear estimator by mimicking the theory of estimators for linear systems. If the system were linear, it is well known that the optimal observer is given by

$$
\Psi(z,(C x-C z))=L(C x-C z),
$$

where $L=\Gamma C^{T} V^{-1}$ and $\Gamma$ solves the ARE for the dual system. Likewise, under certain assumptions, we obtain a suboptimal observer for the nonlinear system by exploiting the dual system. Hence, to find the suboptimal observer, we consider the cost functional

$$
J(\hat{x}, \hat{u})=\frac{1}{2} \int_{0}^{\infty} \hat{x}^{T} U \hat{x}+\hat{u}^{T} V \hat{u} d t
$$

with associated state dynamics given by

$$
\dot{\hat{x}}=A^{T}(\hat{x}) \hat{x}+C^{T}(\hat{x}) \hat{u},
$$

where $U \in \Re^{n \times n}$ is SPSD and $V \in \Re^{m \times m}$ is SPD. Similar to the linear theory, we choose

$$
\Psi\left(x_{e},\left(C(x) x-C\left(x_{e}\right) x_{e}\right)\right)=L\left(x_{e}\right)\left(C(x) x-C\left(x_{e}\right) x_{e}\right),
$$

where

$$
L\left(x_{e}\right)=\Gamma\left(x_{e}\right) C^{T}\left(x_{e}\right) V^{-1}
$$

and $\Gamma\left(x_{e}\right)$ solves the dual SDRE,

$$
\Gamma\left(x_{e}\right) A^{T}\left(x_{e}\right)+A\left(x_{e}\right) \Gamma\left(x_{e}\right)-\Gamma\left(x_{e}\right) C^{T}\left(x_{e}\right) V^{-1} C\left(x_{e}\right) \Gamma\left(x_{e}\right)+U=0 .
$$

The system with the proposed observer is given by

$$
\begin{aligned}
\dot{x} & =A(x) x, \\
\dot{x}_{e} & =A\left(x_{e}\right) x_{e}+L\left(x_{e}\right)\left(y-C\left(x_{e}\right) x_{e}\right), \\
y & =C(x) x .
\end{aligned}
$$

It remains to be shown that the error for the system is asymptotically stable to zero. To develop the theory, we require the following remarks and observations:

Remark 4.1. The observer based SDRE has a solution at each $x$ in the state domain, including $x=0$.

Define $A_{0}=A(0)$ and $C_{0}=C(0)$. Then, the solution at $x=0, \Gamma_{0}$, is the solution to the ARE

$$
\Gamma_{0} A_{0}^{T}+A_{0} \Gamma_{0}-\Gamma_{0} C_{0}^{T} V^{-1} C_{0} \Gamma_{0}+U=0 .
$$

Therefore, each of the state-dependent matrices can be represented by the maps

$$
\begin{aligned}
& A(x)=A_{0}+\Delta A(x), \\
& C(x)=C_{0}+\Delta C(x),
\end{aligned}
$$

and

$$
\Gamma(x)=\Gamma_{0}+\Delta \Gamma(x) .
$$


It follows directly that

$$
L(x)=L_{0}+\Delta L(x)
$$

where

$$
L_{0}=\Gamma_{0} C_{0}^{T} V^{-1}
$$

and

$$
\Delta L(x)=\left(\Delta \Gamma(x) C^{T}(x)+\Gamma_{0} \Delta C^{T}(x)\right) V^{-1} .
$$

Here, $L_{0}$ is the gain matrix that stabilizes $\left(A_{0}^{T}, C_{0}^{T}\right)$. Consequently, $A_{0}-L_{0} C_{0}$ has all eigenvalues with negative real parts. Also, since $\Delta A(x), \Delta C(x)$, and $\Delta \Gamma(x)$ are zero at $x=0$ and continuous, they are small and bounded in a neighborhood of the origin. As a result, $\Delta L(0)=0$ and $\Delta L(x)$ is small in a neighborhood of the origin.

We are now able to prove the following theorem:

Theorem 4.1. Assume that $f(x)$ and $\frac{\partial f(x)}{\partial x_{j}}(j=1, \ldots, n)$ are continuous in $x$ for all $\|x\| \leq \hat{r}$, $\hat{r}>0$ and that $x=0$ is a stable equilibrium point of (41). Additionally assume that $f(x)$ and $y(x)$ can be put into $S D C$ form such that $f(x)=A(x) x$ and $y(x)=C(x) x$ where $(A(x), C(x))$ is a detectable parameterization and $A(x)$ and $C(x)$ are locally Lipschitz for all $x \in \Omega \subseteq \mathcal{B}_{r}(0)$, where $\Omega$ is a nonempty neighborhood of the origin. Then the estimated state given by

$$
\dot{x}_{e}=A\left(x_{e}\right) x_{e}+L\left(x_{e}\right)\left(C(x) x-C\left(x_{e}\right) x_{e}\right),
$$

(where $L(x)$ is given by (39)) will converge locally asymptotically to the state.

Proof. Let $r>0$ be the largest radius such that $\mathcal{B}_{r}(0) \subseteq \Omega$. As in the linear case, we consider the error between the actual state and the estimated state given by

$$
e(t)=x(t)-x_{e}(t)
$$

The error must satisfy the differential equation

$$
\dot{e}(t)=\dot{x}(t)-\dot{x}_{e}(t),
$$

with the initial condition $e_{0}=e(0)=x_{0}-x_{e_{0}}$, where $x_{0}=x(0)$ and $x_{e_{0}}=x_{e}(0)$. Substituting the state (35) and state estimator dynamics (47) into the error dynamics yields,

$$
\begin{aligned}
\dot{e}= & A(x) x-A\left(x_{e}\right) x_{e}-L\left(x_{e}\right)\left(C(x) x-C\left(x_{e}\right) x_{e}\right) \\
= & \left(A_{0}-L_{0} C_{0}\right) e+\Delta A(x) x-\Delta A\left(x_{e}\right) x_{e} \\
& -L\left(x_{e}\right)\left(\Delta C(x) x-\Delta C\left(x_{e}\right) x_{e}\right)-\Delta L\left(x_{e}\right) C_{0} e .
\end{aligned}
$$

We set

$$
\begin{aligned}
g\left(x, x_{e}, e\right)=\Delta A(x) x & -\Delta A\left(x_{e}\right) x_{e} \\
& -L\left(x_{e}\right)\left(\Delta C(x) x-\Delta C\left(x_{e}\right) x_{e}\right) \\
& -\Delta L\left(x_{e}\right) C_{0} e .
\end{aligned}
$$

By the hypotheses that $A(x)$ is locally Lipschitz on $\Omega$, there exists a $\kappa_{A}>0$ such that

$$
\left\|A(x)-A\left(x_{e}\right)\right\| \leq \kappa_{A}\|e\| .
$$

This Lipschitz condition extends to $\Delta A(x)=A(x)-A_{0}$ so that

$$
\left\|\Delta A(x)-\Delta A\left(x_{e}\right)\right\| \leq \kappa_{A}\|e\| .
$$


This, in turn, implies that

$$
\begin{aligned}
&\left\|\Delta A(x) x-\Delta A\left(x_{e}\right) x_{e}\right\|=\| \Delta A(x) x-\Delta A\left(x_{e}\right) x \\
&+\Delta A\left(x_{e}\right) x-\Delta A\left(x_{e}\right) x_{e} \| \\
& \leq\left(\kappa_{A}\|x\|+\left\|\Delta A\left(x_{e}\right)\right\|\right)\|e\| .
\end{aligned}
$$

By the conditions placed on $C(x)$ there exists a Lipschitz constant, $\kappa_{C}$, such that (upon the same manipulation as above)

$$
\left\|\Delta C(x) x-\Delta C\left(x_{e}\right) x_{e}\right\| \leq\left(\kappa_{C}\|x\|+\left\|\Delta C\left(x_{e}\right)\right\|\right)\|e\| .
$$

For notational brevity, we set

$$
\begin{aligned}
h\left(x, x_{e}\right)=\left(\kappa_{A}\|x\|\right. & +\left\|\Delta A\left(x_{e}\right)\right\|+\kappa_{C}\|x\|\left\|L\left(x_{e}\right)\right\| \\
& \left.+\left\|\Delta C\left(x_{e}\right)\right\|\left\|L\left(x_{e}\right)\right\|+\left\|\Delta L\left(x_{e}\right)\right\|\left\|C_{0}\right\|\right)
\end{aligned}
$$

and conclude that

$$
\left\|g\left(x, x_{e}, e\right)\right\| \leq h\left(x, x_{e}\right)\|e\|
$$

By construction of the incremental matrices,

$$
\lim _{x, x_{e} \rightarrow 0} h\left(x, x_{e}\right)=0 .
$$

Due to the detectability condition at the origin and the use of SDRE (and due to construction, LQR) techniques to find the gain, $L_{0}$, there exists $\beta>0$ and $G>0$ such that

$$
\left\|\exp \left(A_{0}-L_{0} C_{0}\right)\right\| \leq G \exp (-\beta t) .
$$

Then, given $\eta \in(0, \beta / G)$ let $\epsilon \in(0, r)$ be such that $h(z, \hat{z}) \leq \eta$ for all $z, \hat{z} \in \mathcal{B}_{\epsilon}(0) \subseteq \Omega$. The equilibrium $x=0$ is stable implying that there exists a $\delta \in(0, \epsilon / 2]$ such that $\|x(t)\|<\epsilon / 2$ for all time so long as $\left\|x_{0}\right\|<\delta$. Let $x_{0}$ and $e_{0}$ be such $x_{0} \in \mathcal{B}_{\delta}(0) \subseteq \Omega$ and $e_{0} \in \mathcal{B}_{\hat{\epsilon}}(0)$, where $\hat{\epsilon}=\epsilon /(2 \hat{G})$ and $\hat{G}=\max \{G, 1\}$. Then the error dynamics have a local solution and are still contained in $\mathcal{B}_{r}(0)$, possibly on only a small interval $[0, \tilde{t})$, and so long as the solution exists it can be expressed by the variations of constants formula

$$
\begin{aligned}
e(t)=\exp \left(\left(A_{0}-\right.\right. & \left.\left.L_{0} C_{0}\right) t\right) e(0) \\
& +\int_{0}^{t} \exp \left(\left(A_{0}-L_{0} C_{0}\right)(t-s)\right) g\left(x(s), x_{e}(s), e(s)\right) d s .
\end{aligned}
$$

By continuity there exists some finite time $\hat{t} \in(0, \tilde{t}]$ such that $e(t)$ is in the ball $\mathcal{B}_{\epsilon / 2}(0)$. Then, upon taking the norm of both sides of (50), we find that for all $t \in[0, \hat{t})$

$$
\|e(t)\| \leq G \exp (-\beta t)\|e(0)\|+G \eta \int_{0}^{t} \exp (-\beta(t-s))\|e(s)\| d s .
$$

We now multiply by $\exp (\beta t)$ and apply the Gronwall inequality so that, for $t \in[0, \hat{t})$,

$$
\exp (\beta t)\|e(t)\| \leq G\left\|e_{0}\right\| \exp (G \eta t)
$$


and it follows that

$$
\|e(t)\| \leq G\left\|e_{0}\right\| \exp ((G \eta-\beta) t) .
$$

By the choice we have made for $e_{0}$ then

$$
\|e(t)\| \leq \frac{\epsilon}{2} \exp ((G \eta-\beta) t) .
$$

But the bound $\eta$ holds only as long as $x_{e}(t)$ remains in $\mathcal{B}_{\epsilon}(0)$. However, because $\left\|x_{e}(t)\right\|-\|x(t)\| \leq$ $\|e(t)\|$,

$$
\left\|x_{e}(t)\right\| \leq \frac{\epsilon}{2}+\frac{\epsilon}{2} \exp ((G \eta-\beta) t) .
$$

Hence, by $\eta<\beta / G, x_{e}$ stays in $\mathcal{B}_{\epsilon}(0)$ for all time and the state estimator converges exponentially to the state.

\subsection{State Estimator Example One}

This example is a simple demonstration of the SDRE technique for state estimation. The system that we wish to observe (from [29]) is given by

$$
\begin{aligned}
& \dot{x}_{1}=x_{2} x_{1}^{2}+x_{2}, \\
& \dot{x}_{2}=-x_{1}^{3}-x_{1}, \\
& y=x_{1} .
\end{aligned}
$$

In SDC form, the above system can be rewritten as

$$
\begin{aligned}
\dot{x} & =\left[\begin{array}{cc}
0 & 1+x_{1}^{2} \\
-\left(1+x_{1}^{2}\right) & 0
\end{array}\right] x \\
y & =\left[\begin{array}{ll}
1 & 0
\end{array}\right] x .
\end{aligned}
$$

The state-dependent observability matrix is

$$
O(x)=\left[\begin{array}{cc}
1 & 0 \\
0 & 1+x_{1}^{2}
\end{array}\right]
$$

Since $O(x)$ has full rank throughout $\Re^{2}$, the system is observable.

We set the weighting matrices in (38) to $U=10 I$ and $V=0.01$. We use the interpolation method to approximate $\Gamma(x)$ where the estimator mesh is $M_{x}=\{-2: 0.1: 2\}$. We use an initial condition of $x_{0}=(1,1)^{T}$ for the state and $x_{e_{0}}=(0.5,0.5)^{T}$ for the estimated state. As a comparison, we also display the state estimator found in [29] given by

$$
\begin{aligned}
& \dot{z}_{1}=-5 z_{1}+z_{2}+5 y+z_{1} z_{2}^{2} \\
& \dot{z}_{2}=-11 z_{1}-z_{1}^{3}+10 y
\end{aligned}
$$

which we refer to as the Thau estimate (for this system). Figures 10(a) and (b) exhibit the fast convergence of each state estimate to $x_{1}$ and $x_{2}$. Figure 10(c) is a state-space representation of the state estimator over time, included as comparison for the plot given in [29]. Figure 10(d) represents the norm of the difference between the actual state and estimated state. We see that the error decreases gradually for the SDRE technique while the error for the Thau state estimator is erratic. 


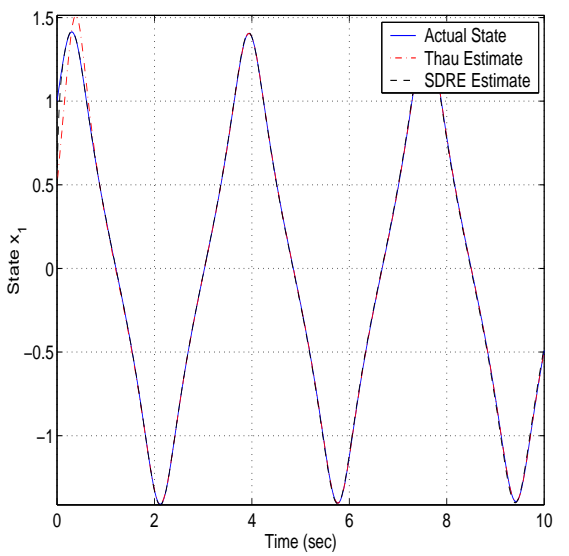

(a)

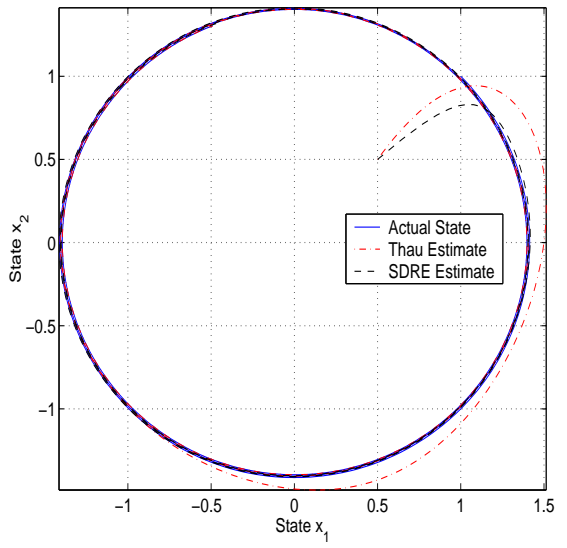

(c)

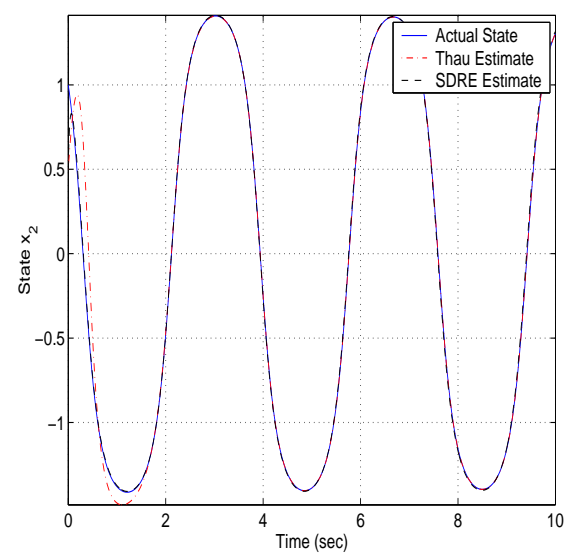

(b)

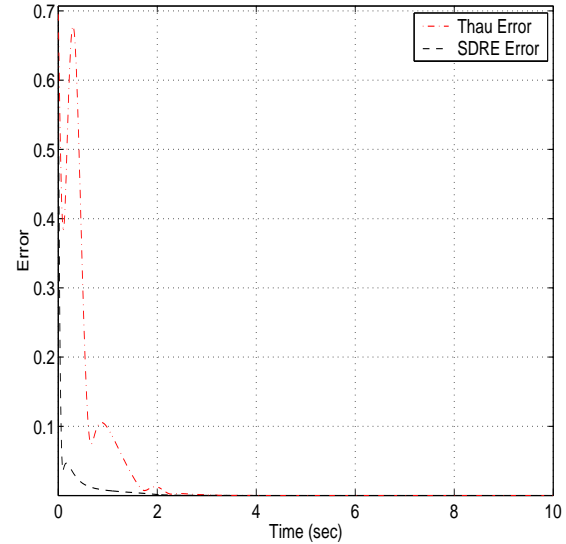

(d)

Figure 10: Plots for Example 4.1, (a) state $x_{1}$ with the Thau and SDRE estimates of $x_{1}$, (b) state $x_{2}$ with the Thau and SDRE estimates of $x_{2}$, (c) state-space representations $x_{2}$ vs. $x_{1}$ for actual state and state estimators, and (d) error of the Thau and SDRE state estimators over the time span $0 \leq t \leq 10$ with state initial condition $x_{0}=(1,1)^{T}$ and state estimator initial condition $x_{e_{0}}=(0.5,0.5)^{T}$. 


\subsection{State Estimator Example Two: Unstable Zero Equilibrium}

We now present an example that shows the effectiveness of the estimator synthesized through the use of SDRE techniques even when the hypotheses of the theorem are not satisfied. Consider the system

$$
\begin{aligned}
& \dot{x}_{1}=x_{2}, \\
& \dot{x}_{2}=-x_{2}\left|x_{2}\right|,
\end{aligned}
$$

with measurement

$$
y=x_{1} .
$$

Lyapunov stability theory states that if there exists a Lyapunov function, $V$, such that in every neighborhood of the origin $V$ and $\dot{V}$ have the same sign, then the origin is unstable. Using the Lyapunov function $V(x)=\|x\|_{2}^{2}$ we see that

$$
\begin{aligned}
\dot{V}(x) & =x_{1} \dot{x}_{1}+x_{2} \dot{x}_{2} \\
& =x_{1} x_{2}-x_{2}^{2}\left|x_{2}\right| .
\end{aligned}
$$

If we let $\epsilon \in(0,1)$ and consider the vector $\hat{x}=(\epsilon / 2, \epsilon / 2)^{T} \in \mathcal{B}_{\epsilon}(0)$. Then, $V(\hat{x})>0$ and since $\epsilon^{2} \leq \epsilon$

$$
\dot{V}(\hat{x})=\frac{\epsilon}{2}\left(\frac{\epsilon}{2}-\frac{\epsilon^{2}}{4}\right)>0 .
$$

Therefore, zero is unstable for the given system and this violates the hypotheses of the theorem.

However, numerically we shall show that the state estimator constructed with SDRE techniques works very well. As a comparison, we consider the following second order estimator from [29] (we will again refer to this as the Thau state estimator):

$$
\begin{aligned}
& \dot{z}_{1}=-20 z_{1}+z_{2}+10 y \\
& \dot{z}_{2}=-100 z_{1}-z_{2}\left|z_{2}\right|+100 y .
\end{aligned}
$$

We can rewrite (56) in the SDC form $f(x)=A(x) x$ where

$$
A(x)=\left[\begin{array}{cc}
0 & 1 \\
0 & -\left|x_{2}\right|
\end{array}\right] .
$$

Since

$$
C=\left[\begin{array}{ll}
1 & 0
\end{array}\right],
$$

the state-dependent observability matrix is $O(x)=I$, so we have that this system is observable for all $x$. For the weighting matrices in (38), we use $U=50 I$ and $V=0.1$. To approximate the solution to the SDRE, we use the interpolation method for $\Pi(x)$ and the mesh used is $M_{x}=\{-5: 0.2: 5\}$. We set the initial condition of the state to $x_{0}=(1,1)^{T}$ and the initial condition of each state estimator to $x_{e_{0}}=(0,0)^{T}$. Figure 11 depicts the behavior of the Thau and SDRE state estimators over a five second time span. Figure 11(a) exhibits the fast convergence of each state estimator to $x_{1}$, where Figure 11(b) conveys that each state estimator takes more time to converge to $x_{2}$. The Thau state estimator is erratic at first and becomes closer to the actual state quicker, whereas the SDRE estimator converges slower but does not stray as far initially. Figure 11(c) is a state-space representation of the state estimator over time, included as comparison for the plot given in [29]. Figure 11(d) represents the norm of the difference of the actual state and estimated state. We see that the error decreases gradually for the SDRE technique while the error for the Thau state estimator increases initially and then decreases abruptly. 


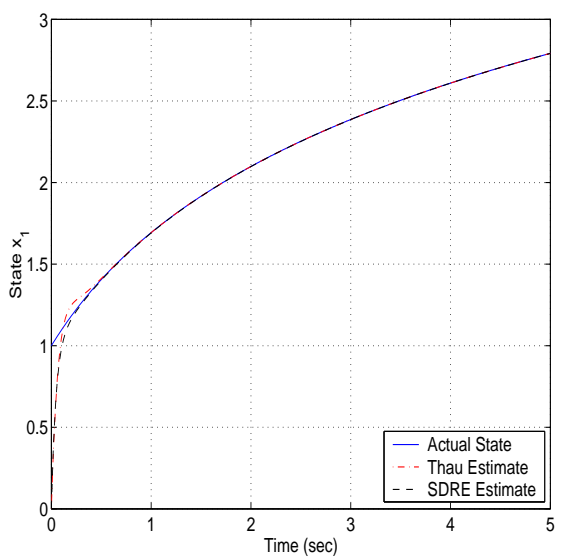

(a)

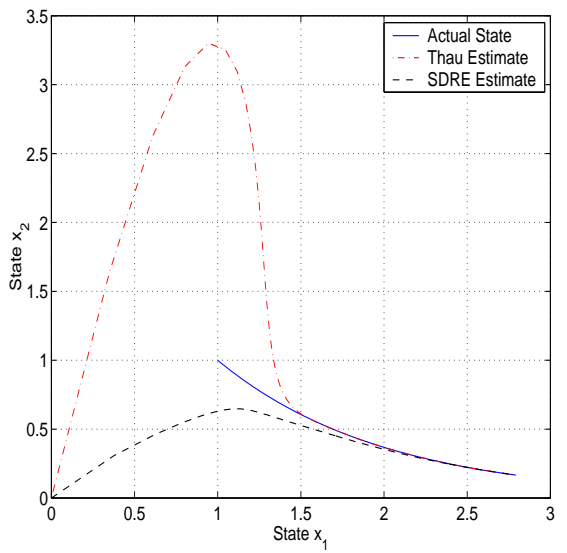

(c)

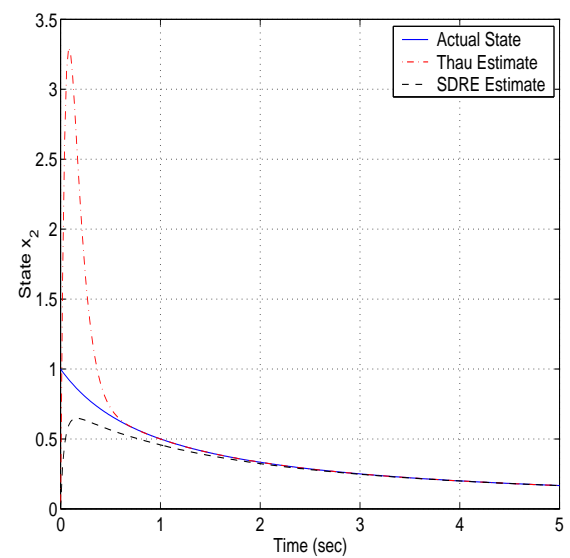

(b)

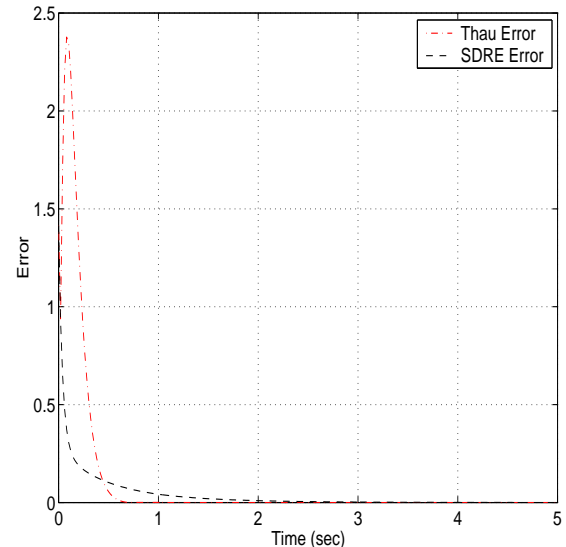

(d)

Figure 11: Plots for Example 4.2, (a) state $x_{1}$ with the Thau and SDRE estimates of $x_{1}$, (b) state $x_{2}$ with the Thau and SDRE estimates of $x_{2}$, (c) state-space representations $x_{2}$ vs. $x_{1}$ for actual state and state estimators, and $(\mathrm{d})$ norm of the error, $\|e(t)\|_{2}$, for the Thau and SDRE state estimators over the time span $0 \leq t \leq 5$ with state initial condition $x_{0}=(1,1)^{T}$ and state estimator initial condition $x_{e_{0}}=(0,0)^{T}$. 


\section{Compensation Using the SDRE State Estimator}

In this section, we investigate the use of the SDRE state estimator in state feedback control laws to compensate given nonlinear systems. We consider systems that can be put in the SDC form

$$
\begin{aligned}
& \dot{x}=A(x) x+B(x) u, \\
& y=C(x) x,
\end{aligned}
$$

where $A(x)$ is a continuous $n \times n$ matrix-valued function, $B(x)$ is a continuous $n \times m$ matrixvalued function and $C(x)$ is a continuous $p \times n$ matrix-valued function. We denote $\Pi(x)$ as the solution to (12), where $Q$ and $R$ are the matrices from the cost functional (1) and $\Gamma(x)$ as the solution to (40), where $U$ and $V$ are the matrices from the cost functional (38). If we again let $L(x)=\Gamma(x) C^{T}(x) V^{-1}$ and $K(x)=R^{-1} B^{T}(x) \Pi(x)$, the control using estimator compensation can be formulated as

$$
\begin{aligned}
\dot{x} & =A(x) x-B(x) K\left(x_{e}\right) x_{e} \\
\dot{x}_{e} & =A\left(x_{e}\right) x_{e}-B\left(x_{e}\right) K\left(x_{e}\right) x_{e}+L\left(x_{e}\right)\left(y-C\left(x_{e}\right) x_{e}\right) \\
y & =C(x) x
\end{aligned}
$$

To show that the compensated system converges asymptotically to zero in a neighborhood about the origin, we require the following remarks:

Remark 5.1. The block matrix

$$
\begin{aligned}
&\left\|\left[\begin{array}{cc}
A & B \\
C & D
\end{array}\right]\right\| \leq\left\|\left[\begin{array}{cc}
A & 0 \\
0 & 0
\end{array}\right]\right\| \\
&+\left\|\left[\begin{array}{ll}
0 & B \\
0 & 0
\end{array}\right]\right\| \\
&+\left\|\left[\begin{array}{ll}
0 & 0 \\
C & 0
\end{array}\right]\right\|+\left\|\left[\begin{array}{ll}
0 & 0 \\
0 & D
\end{array}\right]\right\| \\
&=\|A\|+\|B\|+\|C\|+\|D\|
\end{aligned}
$$

Remark 5.2. The matrix

$$
H(x)=\left[\begin{array}{cc}
A(x)-B(x) K(x) & 0 \\
0 & A(x)-L(x) C(x)
\end{array}\right]
$$

has all eigenvalues with negative real part for all $x$ such that the state-dependent controllability and observability matrices have full rank. This follows directly from the eigenvalue separation property [1], since at each $x, H(x)$ is a constant block diagonal matrix and the blocks each have eigenvalues with real part negative.

We are now able to prove the following theorem for the compensated system:

Theorem 5.1. Assume that the system

$$
\dot{x}=f(x)+B(x) u
$$

is such that $f(x)$ and $\frac{\partial f(x)}{\partial x_{j}}(j=1, \ldots, n)$ are continuous in $x$ for all $\|x\| \leq \hat{r}, \hat{r}>0$, and that $f(x)$ can be written as $f(x)=A(x) x$ (in SDC form). Assume further that $A(x)$ and $B(x)$ are continuous. If $A(x), B(x)$, and $C(x)$ are chosen such that the pair $(A(x), C(x))$ is detectable and $(A(x), B(x))$ is stabilizable for all $x \in \Omega \subseteq \mathcal{B}_{\hat{r}}(0)$ (where $\Omega$ is a nonempty neighborhood of the origin), then $(\hat{x}, \hat{e})=(0,0)$ for system $(57)$ is locally asymptotically stable. Here, $e=x-x_{e}$ is the error between the state and the state estimate in (57). 
Proof. Let $r>0$ be the largest radius such that $\mathcal{B}_{r}(0) \subseteq \Omega$. Using the mapping techniques described in the preceding proofs, one has

$$
\begin{aligned}
A(x) & =A_{0}+\Delta A(x), \\
B(x) & =B_{0}+\Delta B(x), \\
C(x) & =C_{0}+\Delta C(x), \\
K(x) & =K_{0}+\Delta K(x), \\
L(x) & =L_{0}+\Delta L(x),
\end{aligned}
$$

with $L_{0}=L(0)$ and $\Delta L(0)=0$ (etc.) for all of the matrices. The error between the actual state and the estimated state satisfies the differential equation

$$
\dot{e}(t)=\dot{x}(t)-\dot{x}_{e}(t)
$$

with $e(0)=x(0)-x_{e}(0)$. We substitute the state and state estimator dynamics (57) for $\dot{x}$ and $\dot{x}_{e}$ yielding

$$
\begin{aligned}
\dot{e}= & A(x) x-A\left(x_{e}\right) x_{e}-\left(B(x)-B\left(x_{e}\right)\right) K\left(x_{e}\right) x_{e} \\
& -L\left(x_{e}\right)\left(C(x) x-C\left(x_{e}\right) x_{e}\right)
\end{aligned}
$$

As in the theory of linear systems, the dynamics of both $e(t)$ and $x(t)$ are of interest. Hence, the system to be considered is

$$
\left[\begin{array}{l}
x(t) \\
e(t)
\end{array}\right]
$$

with $\dot{x}$ defined in (57) and $\dot{e}$ given by (59). In order to proceed, it is convenient to put the system into the form

$$
\left[\begin{array}{l}
x(t) \\
e(t)
\end{array}\right]=\left[\begin{array}{ll}
H_{11} & H_{12} \\
H_{21} & H_{22}
\end{array}\right]\left[\begin{array}{l}
x(t) \\
e(t)
\end{array}\right]+\left[\begin{array}{ll}
S_{11}\left(x, x_{e}\right) & S_{12}\left(x, x_{e}\right) \\
S_{21}\left(x, x_{e}\right) & S_{22}\left(x, x_{e}\right)
\end{array}\right]\left[\begin{array}{l}
x(t) \\
e(t)
\end{array}\right] .
$$

Rewriting the state dynamics with the given maps, we have that

$$
\dot{x}=\left(A_{0}-B_{0} K_{0}\right) x+\Delta A(x) x-B_{0} \Delta K\left(x_{e}\right) x_{e}-\Delta B(x) K\left(x_{e}\right) x_{e}
$$

To put this in the proper form, we add and subtract the terms $B_{0} \Delta K\left(x_{e}\right) x$ and $\Delta B(x) K\left(x_{e}\right) x$ resulting in

$$
\begin{aligned}
\dot{x}= & \left(A_{0}-B_{0} K_{0}\right) x+\Delta A(x) x-B_{0} \Delta K\left(x_{e}\right) x+B_{0} \Delta K\left(x_{e}\right) e \\
& -\Delta B(x) K\left(x_{e}\right) x+\Delta B(x) K\left(x_{e}\right) e .
\end{aligned}
$$

Thus,

$$
\begin{array}{ll}
H_{11} & =A_{0}-B_{0} K_{0}, \\
S_{11}\left(x, x_{e}\right) & =\Delta A(x)-B_{0} \Delta K\left(x_{e}\right)-\Delta B(x) K\left(x_{e}\right), \\
H_{12} & =0, \\
S_{12}\left(x, x_{e}\right) & =B_{0} \Delta K\left(x_{e}\right)+\Delta B(x) K\left(x_{e}\right) .
\end{array}
$$

Now we seek to formulate the error dynamics in a suitable manner. Rewriting the error dynamics with the given mappings yields

$$
\begin{aligned}
\dot{e}= & \left(A_{0}-L_{0} C_{0}\right) e+\Delta A(x) x-\Delta A\left(x_{e}\right) x_{e} \\
& -\left(\Delta B(x)-\Delta B\left(x_{e}\right)\right) K\left(x_{e}\right) x_{e}-\Delta L\left(x_{e}\right)\left(\Delta C(x) x-\Delta C\left(x_{e}\right) x_{e}\right) \\
& -\Delta L\left(x_{e}\right) C_{0} e-L_{0}\left(\Delta C(x) x-\Delta C\left(x_{e}\right) x_{e}\right)
\end{aligned}
$$


We eliminate all of terms with $x_{e}$ multiples by adding and subtracting terms (if the term consists of a matrix times $x_{e}$ we add and subtract that matrix times $x$ ). This leaves us with

$$
\begin{aligned}
\dot{e}= & \left(A_{0}-L_{0} C_{0}\right) e+\Delta A(x) x+\Delta A\left(x_{e}\right) e-\Delta A\left(x_{e}\right) x \\
& -\left(\Delta B(x)-\Delta B\left(x_{e}\right)\right) K\left(x_{e}\right) x+\left(\Delta B(x)-\Delta B\left(x_{e}\right)\right) K\left(x_{e}\right) e \\
& -\Delta L\left(x_{e}\right) \Delta C(x) x+\Delta L\left(x_{e}\right) \Delta C\left(x_{e}\right) x-\Delta L\left(x_{e}\right) \Delta C\left(x_{e}\right) e \\
& -\Delta L\left(x_{e}\right) C_{0} e-L_{0} \Delta C(x) x+L_{0} \Delta C\left(x_{e}\right) x-L_{0} \Delta C\left(x_{e}\right) e .
\end{aligned}
$$

Thus, we have manipulated the error dynamics as desired and we have that

$$
\begin{aligned}
H_{21} & =0 \\
S_{21}\left(x, x_{e}\right)= & \Delta A(x)-\Delta A\left(x_{e}\right)-\Delta B(x) K\left(x_{e}\right)+\Delta B\left(x_{e}\right) K\left(x_{e}\right) \\
& -\Delta L\left(x_{e}\right) \Delta C(x)+\Delta L\left(x_{e}\right) \Delta C\left(x_{e}\right)-L_{0} \Delta C(x) \\
& +L_{0} \Delta C\left(x_{e}\right), \\
H_{22} & A_{0}-L_{0} C_{0}, \\
S_{22}\left(x, x_{e}\right)= & \Delta A\left(x_{e}\right)+\Delta B(x) K\left(x_{e}\right)-\Delta B\left(x_{e}\right) K\left(x_{e}\right) \\
& -\Delta L\left(x_{e}\right) \Delta C\left(x_{e}\right)-\Delta L\left(x_{e}\right) C_{0}-L_{0} \Delta C\left(x_{e}\right) .
\end{aligned}
$$

Let

$$
\bar{H}=\left[\begin{array}{ll}
H_{11} & H_{12} \\
H_{21} & H_{22}
\end{array}\right]
$$

and

$$
\bar{S}\left(x, x_{e}\right)=\left[\begin{array}{ll}
S_{11}\left(x, x_{e}\right) & S_{12}\left(x, x_{e}\right) \\
S_{21}\left(x, x_{e}\right) & S_{22}\left(x, x_{e}\right)
\end{array}\right] .
$$

By Remark 5.1, we can bound the matrix norm by

$$
\left\|\bar{S}\left(x, x_{e}\right)\right\| \leq\left\|S_{11}\left(x, x_{e}\right)\right\|+\left\|S_{12}\left(x, x_{e}\right)\right\|+\left\|S_{21}\left(x, x_{e}\right)\right\|+\left\|S_{22}\left(x, x_{e}\right)\right\| .
$$

Then, taking the norm of each of these matrices, we find

$$
\begin{aligned}
\left\|S_{11}\left(x, x_{e}\right)\right\| \leq & \|\Delta A(x)\|+\left\|B_{0}\right\|\left\|\Delta K\left(x_{e}\right)\right\|+\|\Delta B(x)\|\left\|K\left(x_{e}\right)\right\|, \\
\left\|S_{21}\left(x, x_{e}\right)\right\| \leq & \|\Delta A(x)\|+\left\|\Delta A\left(x_{e}\right)\right\|+\|\Delta B(x)\|\left\|K\left(x_{e}\right)\right\| \\
& +\left\|\Delta B\left(x_{e}\right)\right\|\left\|K\left(x_{e}\right)\right\|+\left\|\Delta L\left(x_{e}\right)\right\|\|\Delta C(x)\| \\
& +\left\|\Delta L\left(x_{e}\right)\right\|\left\|\Delta C\left(x_{e}\right)\right\|+\left\|L_{0}\right\|\|\Delta C(x)\|+\left\|L_{0}\right\|\left\|\Delta C\left(x_{e}\right)\right\|, \\
\left\|S_{12}\left(x, x_{e}\right)\right\| \leq & \left\|B_{0}\right\|\left\|\Delta K\left(x_{e}\right)\right\|+\|\Delta B(x)\|\left\|K\left(x_{e}\right)\right\|, \\
\left\|S_{22}\left(x, x_{e}\right)\right\| \leq & \left\|\Delta A\left(x_{e}\right)\right\|+\|\Delta B(x)\|\left\|K\left(x_{e}\right)\right\|+\left\|\Delta B\left(x_{e}\right)\right\|\left\|K\left(x_{e}\right)\right\| \\
& +\left\|\Delta L\left(x_{e}\right)\right\|\left\|\Delta C\left(x_{e}\right)\right\|+\left\|\Delta L\left(x_{e}\right)\right\|\left\|C_{0}\right\| \\
& +\left\|L_{0}\right\|\left\|\Delta C\left(x_{e}\right)\right\| .
\end{aligned}
$$

Therefore,

$$
\begin{aligned}
\left\|\bar{S}\left(x, x_{e}\right)\right\| \leq & 2\left(\|\Delta A(x)\|+\left\|\Delta A\left(x_{e}\right)\right\|\right)+2\left\|B_{0}\right\|\left\|\Delta K\left(x_{e}\right)\right\| \\
& +2\left\|K\left(x_{e}\right)\right\|\left(2\|\Delta B(x)\|+\left\|\Delta B\left(x_{e}\right)\right\|\right)+2\left\|\Delta L\left(x_{e}\right)\right\|\left\|\Delta C\left(x_{e}\right)\right\| \\
& +\left\|\Delta L\left(x_{e}\right)\right\|\|\Delta C(x)\|+\left\|L_{0}\right\|\left(2\left\|\Delta C\left(x_{e}\right)\right\|+\|\Delta C(x)\|\right) \\
& +\left\|C_{0}\right\|\left\|\Delta L\left(x_{e}\right)\right\| \\
= & g\left(x, x_{e}\right) .
\end{aligned}
$$

By definition of the incremental matrices, as $x, x_{e} \rightarrow 0, g\left(x, x_{e}\right) \rightarrow 0$. Thus, for any $\eta>0$ there exists an $\alpha \in(0, r)$ so that if $z, \hat{z} \in \mathcal{B}_{\alpha}(0)$ then

$$
\|\bar{S}(z, \hat{z})\| \leq \eta
$$


Using the variations of constants formula with nonhomogeneous part

$$
\bar{S}\left(x, x_{e}\right)\left[\begin{array}{l}
x \\
e
\end{array}\right],
$$

we have that the solution for the system (so long as it exists) is given by

$$
\left[\begin{array}{l}
x(t) \\
e(t)
\end{array}\right]=\exp (\bar{H} t)\left[\begin{array}{l}
x_{0} \\
e_{0}
\end{array}\right]+\int_{0}^{t} \exp (\bar{H}(t-s)) \bar{S}\left(x, x_{e}\right)\left[\begin{array}{l}
x(s) \\
e(s)
\end{array}\right] d s .
$$

If we assume that

$$
\left[\begin{array}{l}
x_{0} \\
e_{0}
\end{array}\right] \in \mathcal{B}_{\alpha / 2}(0)
$$

then $x_{0} \in \mathcal{B}_{\alpha / 2}(0)$ and $e_{0} \in \mathcal{B}_{\alpha / 2}(0)$ (which implies that $\left.x_{e_{0}} \in \mathcal{B}_{\alpha}(0)\right)$ so that

$$
\left\|\bar{S}\left(x, x_{e}\right)\right\| \leq \eta
$$

Then, for as long as

$$
\left\|\left[\begin{array}{l}
x(t) \\
e(t)
\end{array}\right]\right\| \leq \frac{\alpha}{2}
$$

the inequality

$$
\left\|\left[\begin{array}{l}
x(t) \\
e(t)
\end{array}\right]\right\| \leq\|\exp (\bar{H} t)\|\left\|\left[\begin{array}{l}
x_{0} \\
e_{0}
\end{array}\right]\right\|+\eta \int_{0}^{t}\|\exp (\bar{H}(t-s))\|\left\|\left[\begin{array}{c}
x(s) \\
e(s)
\end{array}\right]\right\| d s
$$

holds.

By the eigenvalue separation principle (see Remark 5.2), there exists a constant $\beta>0$ such that

$$
\text { real }\left\{\operatorname{eigs}\left(\left[\begin{array}{cc}
A_{0}-B_{0} K_{0} & 0 \\
0 & A_{0}-L_{0} C_{0}
\end{array}\right]\right)\right\}<-\beta .
$$

We know there also exists a $G>0$ such that

$$
\|\exp (\bar{H} t)\| \leq G \exp (-\beta t) .
$$

Let $\hat{t}$ represent the amount of time that

$$
\left[\begin{array}{l}
x(t) \\
e(t)
\end{array}\right] \in \mathcal{B}_{\alpha / 2}(0)
$$

Then, for $t \in[0, \hat{t})$ the state and error dynamics are bounded by

$$
\left\|\left[\begin{array}{l}
x(t) \\
e(t)
\end{array}\right]\right\| \leq G \exp (-\beta t)\left\|\left[\begin{array}{l}
x_{0} \\
e_{0}
\end{array}\right]\right\|+G \eta \int_{0}^{t} \exp (-\beta(t-s))\left\|\left[\begin{array}{l}
x(s) \\
e(s)
\end{array}\right]\right\| d s .
$$

Upon multiplying both sides by $\exp (\beta t)$, we have the relation

$$
\exp (\beta t)\left\|\left[\begin{array}{l}
x(t) \\
e(t)
\end{array}\right]\right\| \leq G\left\|\left[\begin{array}{l}
x_{0} \\
e_{0}
\end{array}\right]\right\|+G \eta \int_{0}^{t} \exp (\beta s)\left\|\left[\begin{array}{c}
x(t) \\
e(t)
\end{array}\right]\right\| d s .
$$

and invoking the Gronwall inequality we obtain

$$
\left\|\left[\begin{array}{l}
x(t) \\
e(t)
\end{array}\right]\right\| \exp (\beta t) \leq\left\|\left[\begin{array}{l}
x_{0} \\
e_{0}
\end{array}\right]\right\| G \exp (G \eta t) .
$$


Multiplying through each side by $\exp (-\beta t)$, we thus find that

$$
\left\|\left[\begin{array}{l}
x(t) \\
e(t)
\end{array}\right]\right\| \leq\left\|\left[\begin{array}{l}
x_{0} \\
e_{0}
\end{array}\right]\right\| G \exp (-(\beta-G \eta) t)
$$

for all $t \in[0, \hat{t})$. Recall that the bound $\eta$ holds true so long as the trajectories of both $x(t)$ and $x_{e}(t)$ remain in an $\alpha$-ball of the origin. Thus, we must also consider the bound placed upon $x_{e}(t)$. Using the inequality

$$
\left\|x_{e}(t)\right\|-\|x(t)\| \leq\|e(t)\| \leq\left\|\left[\begin{array}{l}
x(t) \\
e(t)
\end{array}\right]\right\|
$$

we obtain

$$
\begin{aligned}
\left\|x_{e}(t)\right\| & \leq\|x(t)\|+\left\|\left[\begin{array}{l}
x_{0} \\
e_{0}
\end{array}\right]\right\| G \exp (-(\beta-G \eta) t) \\
& \leq 2\left\|\left[\begin{array}{l}
x_{0} \\
e_{0}
\end{array}\right]\right\| G \exp (-(\beta-G \eta) t) .
\end{aligned}
$$

To this point, $\eta$ was an arbitrary constant. However, if we set $0<\eta<\beta / G$ and let $\epsilon \in(0, \alpha]$ (where $\alpha$ corresponds to this specific $\eta$ ) be given we find that so long as

$$
\left[\begin{array}{l}
x_{0} \\
e_{0}
\end{array}\right] \in \mathcal{B}_{\delta}(0)
$$

with $\delta=\epsilon /(2 \hat{G})($ where $\hat{G}=\max \{1, G\})$, then

$$
\left\|\left[\begin{array}{l}
x(t) \\
e(t)
\end{array}\right]\right\| \leq \frac{\epsilon}{2}
$$

and

$$
\left\|x_{e}(t)\right\| \leq \epsilon
$$

so that both bounds are for all $t$. Since the solution can be continued to the boundary (and hence, to $\left.\mathcal{B}_{r}(0)\right)$ and the solution is bounded by (61) where $\beta-G \eta>0$, we can conclude that the origin is asymptotically stable.

\subsection{Compensation Example}

This example is a simple demonstration of the SDRE technique for the control of a system using state estimator based compensation. We demonstrate the effectiveness of the compensated system by adding a control term to Example 4.1. Hence, the system is

$$
\begin{aligned}
& \dot{x}_{1}=x_{2} x_{1}^{2}+x_{2}+u, \\
& \dot{x}_{2}=-x_{1}^{3}-x_{1}, \\
& y=x_{1} .
\end{aligned}
$$

In SDC form, the above system, along with an SDRE compensator, can be rewritten as

$$
\begin{aligned}
& \dot{x}=\left[\begin{array}{cc}
0 & 1+x_{1}^{2} \\
-\left(1+x_{1}^{2}\right) & 0
\end{array}\right] x+\left[\begin{array}{l}
1 \\
0
\end{array}\right] u\left(x_{e}\right) \\
& \dot{x}_{e}=\left[\begin{array}{cc}
0 & 1+x_{e_{1}}^{2} \\
-\left(1+x_{e_{1}}^{2}\right) & 0
\end{array}\right] x_{e}+\left[\begin{array}{l}
1 \\
0
\end{array}\right] u\left(x_{e}\right)+L\left(x_{e}\right)\left(y-x_{e_{1}}\right) \\
& y=\left[\begin{array}{ll}
1 & 0
\end{array}\right] x .
\end{aligned}
$$


The state-dependent controllability matrix for this system is

$$
M(x)=\left[\begin{array}{cc}
1 & 0 \\
0 & -\left(1+x_{1}^{2}\right)
\end{array}\right]
$$

and the state-dependent observability matrix is

$$
O(x)=\left[\begin{array}{cc}
1 & 0 \\
0 & 1+x_{1}^{2}
\end{array}\right]
$$

Since both $M(x)$ and $O(x)$ have full rank throughout $\Re^{2}$, the system is controllable and observable.

For the weighting matrices in (1) and (38) we use $Q=5 I, R=1, U=10 I$, and $V=0.01$. The interpolation method provides a nice approach to approximate both $\Pi(x)$ and $\Gamma(x)$. The state estimator mesh and controller mesh are the same, $M_{x}=\{-2: 0.1: 2\}$. We use an initial condition of $x_{0}=(1,1)^{T}$ for the state and $x_{e_{0}}=(0.5,0.5)^{T}$ for the estimated state and turn the controller on at $t=2.0$ seconds. Figures 12 (a) and 12(b) depict the uncontrolled, controlled, and estimated states for this example. We find that the estimator converges to the state quickly and the controller stabilizes the system. Figure 12(c) is the state-space representation, while Figure 12(d) is the error over the time interval and Figure 12(e) exhibits the control effort using state estimator based compensation. 


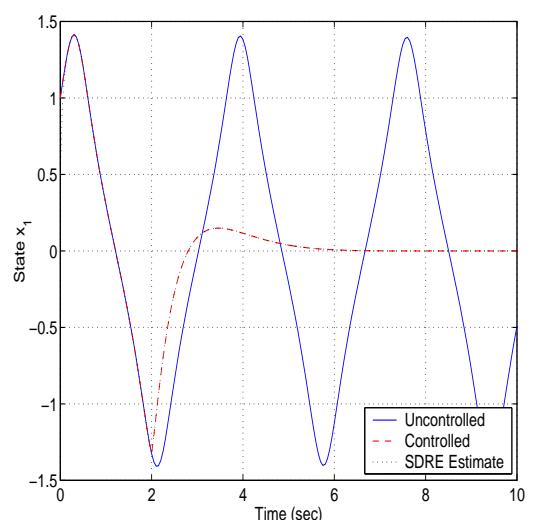

(a)

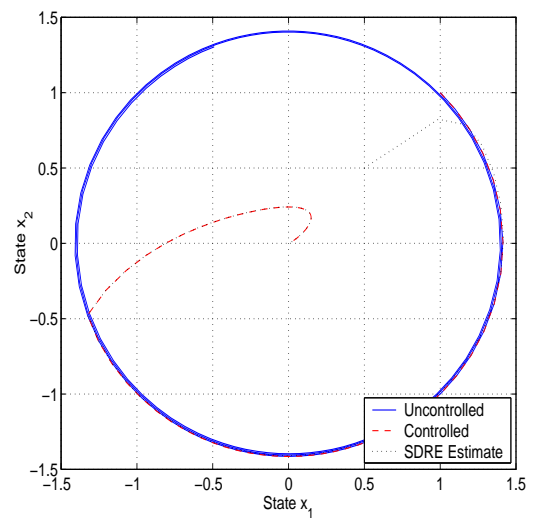

(c)

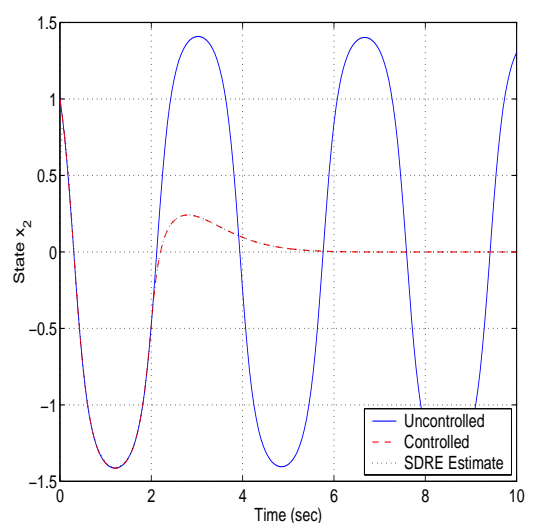

(b)

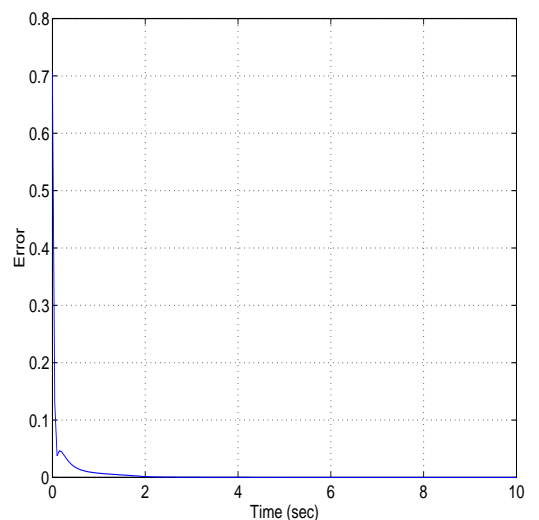

(d)

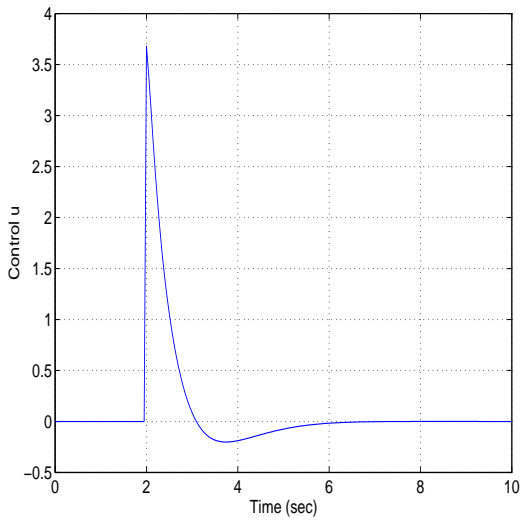

(e)

Figure 12: Plots for Example 5.1, (a) state $x_{1}$, (b) state $x_{2}$, and (c) state-space representations for uncontrolled, controlled, and estimated $\left(x_{e}(t)\right)$ systems, (d) norm of the error $\|e(t)\|_{2}$, and (e) compensated control for the system over the time span $0 \leq t \leq 5$ with state initial condition $x_{0}=(1,1)^{T}$ and state estimator initial condition $x_{e_{0}}=(0.5,0.5)^{T}$. 


\section{Conclusion}

In this paper we have considered SDRE techniques for the general design and synthesis of feedback controllers, estimators, and compensators of nonlinear systems. In particular, the SDRE methods were formulated for cost functionals with constant weighting coefficients. The resulting closed loop system was shown to be locally asymptotically stable and a local convergent result was obtained for the nonlinear state estimator. In addition, two approaches were presented for the numerical approximation of the solution to the SDRE for a large class of nonlinear problems. The numerical approach is based on interpolation of SDRE solutions or controls over the state space. This approach is very easy to implement and was shown to perform very well on a wide class of nonlinear systems.

\section{Acknowledgements}

Research reported here was supported in part by the U.S. Air Force Office of Scientific Research under grant AFOSR F49620-01-1-0026 and in part by the Joint DMS/NIGMS Initiative to Support Research in the Area of Mathematical Biology under grant 1R01GM67299-01.

\section{References}

[1] B. D. O. Anderson and J. B. Moore. Optimal Control Linear Quadratic Methods. Prentice Hall, Englewood Cliffs, New Jersey, 1990.

[2] H. T. Banks, S. C. Beeler, G. M. Kepler, and H. T. Tran. Feedback control of thin film growth in an HPCVD reactor via reduced order models. In Proceedings of the 40th IEEE Conference on Decision and Control, Orlando, Florida, 2001.

[3] H. T. Banks, S. C. Beeler, G. M. Kepler, and H. T. Tran. Reduced order modeling and control of thin film growth in an HPCVD reactor. SIAM J. Applied Mathematics, 62(4):1251-1280, 2002. CRSC Technical Report CRSC-TR00-33, NCSU.

[4] H. T. Banks, D. M. Bortz, and S. E. Holte. Incorporation of variability into the modeling of viral delays in HIV infection dynamics. Mathematical Biosciences, 183:63-91, 2003. CRSC Technical Report CRSC-TR01-25, NCSU.

[5] S. C. Beeler, H. T. Tran, and H. T. Banks. Feedback control methodologies for nonlinear systems. Journal of Optimization Theory and Applications, 107(1):1-33, 2000.

[6] S. C. Beeler, H. T. Tran, and H. T. Banks. State estimation and tracking control of nonlinear dynamical systems. In W. Desch, F. Kappel, and K. Kunisch, editors, Control and Estimation of Distributed Parameter Systems, volume 143 of International Series of Numerical Mathematics, pages 1-24. Birkhäuser, 2002. CRSC Technical Report CRSC-TR00-19, NCSU.

[7] S. C. Beeler. Modeling and Control of Thin Film Growth in a Chemical Vapor Deposition Reactor. Ph.d. dissertation, North Carolina State University, Raleigh, North Carolina, Dec. 2000 .

[8] F. Brauer and J. A. Nohel. The Qualitative Theory of Ordinary Differential Equations. Dover Publications, Mineola, New York, 1989. 
[9] J. R. Cloutier, C. N. D'Souza, and C. P. Mracek. Nonlinear regulation and nonlinear $h_{\infty}$ control via the state-dependent riccati equation technique: Part 1. theory. In Proceedings of the First International Conference on Nonlinear Problems in Aviation and Aerospace, Daytona Beach, Florida, 1996.

[10] J. R. Cloutier, C. P. Mracek, D. B. Ridgely, and K. D. Hammett. State-dependent riccati equation techniques: Theory and applications. In Notes from the SDRE Workshop conducted at the American Control Conference, Philadelphia, Pennsylvania, 1998.

[11] J. Doyle, Y. Huang, J. Primbs, R. Freeman, R. Murray, A. Packard, and M. Krstic. Nonlinear control: Comparisons and case studies. In Notes from the Nonlinear Control Workshop conducted at the American Control Conference, Albuquerque, New Mexico, 1998.

[12] B. Friedland. Advanced Control System Design. Prentice Hall, Englewood Cliffs, New Jersey, 1996.

[13] R. A. Hull, J. R. Cloutier, C. P. Mracek, and D. T. Stansbery. State-dependent riccati equation solution of the toy nonlinear optimal control problem. In Proceedings of the American Control Conference, Philadelphia, Pennsylvania, 1998.

[14] X. Hu. On state observers for nonlinear systems. Systems and Control Letters, 17:465-473, 1991.

[15] A. Isidori. Nonlinear Control Systems. Springer-Verlag, New York, New York, 1995.

[16] K. Ito and J. D. Schroeter. Reduced order feedback synthesis for viscous incompressible flows. Mathematical and Computer Modeling, 33:173-192, 2001.

[17] D. Kirschner. Using mathematics to understand HIV immune dynamics. AMS Notices, pages 191-202, Feb. 1996.

[18] M. Krstic, I. Kanellakopoulos, and P. Kokotovic. Nonlinear and Adaptive Control Design. John Wiley and Sons, New York, New York, 1995.

[19] F. L. Lewis and V. L. Syrmos. Optimal Control. John Wiley and Sons, New York, New York, 1995.

[20] J. Markman and I. N. Katz. An iterative algorithm for solving hamilton jacobi type equations. SIAM Journal on Scientific Computing, 22(1):312-329, 2000.

[21] C. P. Mracek and J. R. Cloutier. Control designs for the nonlinear benchmark problem via the state-dependent riccati equation method. International Journal of Robust and Nonlinear Control, 8:401-433, 1998.

[22] D. K. Parrish and D. B. Ridgely. Attitude control of a satellite using the SDRE method. In Proceedings of the American Control Conference, Albuquerque, New Mexico, 1997.

[23] D. K. Parrish and D. B. Ridgely. Control of an artificial human pancreas using the SDRE method. In Proceedings of the American Control Conference, Albuquerque, New Mexico, 1997.

[24] L. Rodman. On extremal solutions of the algebraic riccati equation. In C. I. Byrnes and C. F. Martin, editors, Algebraic and Geometric Methods in Linear Systems Theory, volume 18 of Lectures in Applied Mathematics. American Mathematical Society, Providence, Rhode Island, 1980. 
[25] J. S. Shamma and M. Athens. Analysis of gain scheduled control for nonlinear plants. IEEE Trans. on Auto. Control, 35(8):898-907, 1990.

[26] J. S. Shamma and J. R. Cloutier. Existence of SDRE stabilizing feedback. In Proceedings of the American Control Conference, Arlington, Virginia, 2001.

[27] J.-J. E. Slotine. Applied nonlinear control. Prentice Hall, Englewood Cliffs, New Jersey, 1991.

[28] D. T. Stansbery and J. R. Cloutier. Position and attitude control of a spacecraft using the statedependent riccati equation technique. In Proceedings of the American Control Conference, Chicago, Illinois, 2000.

[29] F. E. Thau. Observing the state of non-linear dynamic systems. International Journal of Control, 17:471-479, 1973.

[30] A. Theodoropoulou, R. A. Adomaitis, and E. Zafiriou. Model reduction for optimization of rapid thermal chemical vapor deposition systems. IEEE Transactions on Semiconductor Manufacturing, 11:85-98, 1998.

[31] L. C. To, M. O. Tade, and M. Kraetzl. Robust Nonlinear Control of Industrial Evaporation Systems. World Scientific, River Edge, New Jersey, 1999.

[32] A. Wernli and G. Cook. Suboptimal control for the nonlinear quadratic regulator problem. Automatica, 11:75-84, 1975.

[33] K. Zhou, J. Doyle, and K. Glover. Robust and Optimal Control. Prentice Hall, Englewood Cliffs, New Jersey, 1996. 